\title{
Synthesis and Evaluation of Coumermycin A1 Analogues that Inhibit the Hsp90 Protein Folding Machinery
}

\author{
Joseph A. Burlison and Brian S. J. Blagg* \\ Supporting information
}

Department of Medicinal Chemistry, The University of Kansas, 1251 Wescoe Hall Drive, Malott 4070, Lawrence, Kansas 66045-7563

bblagg@ku.edu 
Table of Contents

Experimental for Compound $5 \quad$ S3

Experimental for Compound $6 \quad$ S3

Experimental for Compound $7 \quad$ S3

Experimental for Compound $8 \quad \mathrm{~S} 4$

Experimental for Compound $\mathbf{9} \quad \mathrm{S} 4$

Experimental for Compound $\mathbf{1 0} \quad$ S5

Experimental for Compound $\mathbf{1 5} \quad$ S5

Experimental for Compound $\mathbf{1 6} \quad$ S6

Experimental for Compound $\mathbf{1 7} \quad$ S6

Experimental for Compound $\mathbf{1 8} \quad$ S6

$\begin{array}{ll}\text { Experimental for Compound 15a } & \text { S7 }\end{array}$

$\begin{array}{ll}\text { Experimental for Compound 16a } & \text { S7 }\end{array}$

Experimental for Compound 17a $\quad$ S8

Experimental for Compound 18a $\quad$ S8

Experimental for Compound $23 \quad$ S8

Experimental for Compound $24 \quad$ S9

Experimental for Compound $25 \quad$ S9

Experimental for Compound $26 \quad$ S10

Experimental for Compound 27 $\quad$ S11

Experimental for Compound $28 \quad$ S11

Experimental for Compound $29 \quad$ S11

Experimental for Compound $\mathbf{3 0} \quad$ S11

Experimental for Compound $31 \quad \mathrm{~S} 12$

Experimental for Compound $32 \quad \mathrm{~S} 12$

Experimental for Compound $33 \quad \mathrm{~S} 13$

$\begin{array}{ll}\text { Experimental for Compound } 35 & \mathrm{~S} 13\end{array}$

$\begin{array}{ll}\text { Experimental for Compound } 36 & \mathrm{~S} 14\end{array}$

$\begin{array}{ll}\text { Experimental for Compound 37a } & \mathrm{S} 14\end{array}$

$\begin{array}{ll}\text { Experimental for Compound 38a } & \text { S14 }\end{array}$

$\begin{array}{ll}\text { Experimental for Compound } 37 & \mathrm{~S} 15\end{array}$

$\begin{array}{ll}\text { Experimental for Compound } 38 & \text { S15 }\end{array}$

Experimental for Compound $\mathbf{4 1} \quad \mathrm{S} 16$

Experimental for Compound $42 \quad \mathrm{~S} 16$

$\begin{array}{ll}\text { References } & \text { S17 }\end{array}$ 
Benzyl-7-((3aR, $\quad 4 R, \quad 7 R, \quad 7 \mathrm{a})$-7-methoxy-6,6-dimethyl-2-oxotetrahydrdo-3aH[1.3]dioxolo[4,5-c]pyran-4-yloxy)-8-methyl-2-oxo-2H-chromen-3-ylcarbamate (5). Boron trifluoride etherate $(61 \mu \mathrm{L}, 69 \mathrm{mg}, 0.49 \mathrm{mmol}, 30 \mathrm{~mol} \%)$ was added dropwise to a solution of (3aR, 4S, 7R, 7aR)-7methoxy-6,6-dimethyl-2-oxo-tetrahydro-3aH-[1,3]dioxolo[4,5-c]pyran-4-yl 2,2,2-trichloroacetimidate ${ }^{1,2}$ (4, $588 \mathrm{mg}, 1.62 \mathrm{mmol}$ ) and benzyl 7-hydroxy-8-methyl-2-oxo-2H-chromen-3-yl carbamate ${ }^{3}$ (3, $527 \mathrm{mg}, 1.62$ $\mathrm{mmol})$ in $\mathrm{CH}_{2} \mathrm{Cl}_{2}(16 \mathrm{~mL})$. The reaction mixture stirred for $14 \mathrm{~h}$, before three drops of $\mathrm{Et}_{3} \mathrm{~N}$ was added and the mixture concentrated. The residue was purified by chromatography $\left(\mathrm{SiO}_{2}, \mathrm{DCM} \rightarrow 100: 1\right.$; $\mathrm{CH}_{2} \mathrm{Cl}_{2}$ :acetone) to afford 5 (670 mg, 81\%) as a yellow foam: $[\alpha]^{22}{ }_{\mathrm{D}}=-19.7^{\circ}(\mathrm{c}=1.54,20 \% \mathrm{MeOH}$ in $\left.\mathrm{CH}_{2} \mathrm{Cl}_{2}\right) ;{ }^{1} \mathrm{H}$ NMR $\left(\mathrm{CDCl}_{3}, 400 \mathrm{MHz}\right) \delta 8.27(\mathrm{~s}, 1 \mathrm{H}), 7.85(\mathrm{~s}, 1 \mathrm{H}), 7.55-7.35(\mathrm{~m}, 5 \mathrm{H}), 7.29(\mathrm{~d}, J=2.9 \mathrm{~Hz}$, 1H), 7.11 (d, $J=8.7 \mathrm{~Hz}, 1 \mathrm{H}), 5.77$ (d, $J=1.9 \mathrm{~Hz}, 1 \mathrm{H}), 5.23$ (s, 2H), 5.05 (d, $J=1.9 \mathrm{~Hz}, 1 \mathrm{H}), 4.95$ (t, $J=$ $7.7 \mathrm{~Hz}, 1 \mathrm{H}), 3.59$ (s, 3H), $3.30(\mathrm{~d}, J=7.6 \mathrm{~Hz}, 1 \mathrm{H}), 2.27$ (s, 3H), $1.34(\mathrm{~s}, 3 \mathrm{H}), 1.19(\mathrm{~s}, 3 \mathrm{H}) ;{ }^{13} \mathrm{C} \mathrm{NMR}$ $\left(\mathrm{CDCl}_{3}, 100 \mathrm{MHz}\right) \delta 159.0,155.2,153.6,153.6,149.2,136.0,129.1$ (2C), 129.0, 128.7 (2C), 125.8, 122.6, 122.1, 115.2, 115.1, 111.6, 94.8, 83.3, 78.4, 77.6, 77.0, 67.9, 61.0, 27.9, 22.6, 8.8; IR (film) $v_{\max } 3402$, $3319,3063,3034,2984,2939,2839,1817,1709,1634,1609,1587,1522,1456,1383,1366,1331,1296$, 1263, 1229, 1205, $1175 \mathrm{~cm}^{-1}$; HRMS (ESI+) $\mathrm{m} / \mathrm{z} 526.1688\left(\mathrm{M}+\mathrm{H}^{+}, \mathrm{C}_{27} \mathrm{H}_{28} \mathrm{NO}_{10}\right.$ requires $\left.\mathrm{m} / \mathrm{z} 526.1713\right)$.

6

3-Amino-7-((3aR, 4R, 7R, 7aR)-7-methoxy-6,6-dimethyl-2-oxo oxotetrahydrdo-3aH[1.3]dioxolo[4,5-c]pyran-4-yloxy)-8-methyl-2H-chromen-2-one (6). Palladium on carbon (10\%, $67 \mathrm{mg})$ was added to a solution of carbamate $5(670 \mathrm{mg}, 1.31 \mathrm{mmol})$ in THF $(13 \mathrm{~mL})$. The suspension was stirred for $6 \mathrm{~h}$ under a hydrogen atmosphere and was filtered through a plug of $\mathrm{SiO}_{2}$. The solvent was removed and the residue purified by chromatography $\left(\mathrm{SiO}_{2}, 100: 1 \rightarrow 50: 1 ; \mathrm{CH}_{2} \mathrm{Cl}_{2}\right.$ :acetone) to afford 6 (425 mg, 83\%) as a pale yellow foam: $[\alpha]^{23}{ }_{\mathrm{D}}=-26.4^{\circ}\left(\mathrm{c}=0.780,20 \% \mathrm{MeOH}\right.$ in $\left.\mathrm{CH}_{2} \mathrm{Cl}_{2}\right) ;{ }^{1} \mathrm{H}$ NMR $\left(\mathrm{CDCl}_{3}, 400\right.$ MHz) $\delta 7.10(\mathrm{~d}, J=8.6 \mathrm{~Hz}, 1 \mathrm{H}), 7.05(\mathrm{~d}, J=8.6 \mathrm{~Hz}, 1 \mathrm{H}), 6.68(\mathrm{~s}, 1 \mathrm{H}), 5.73(\mathrm{~d}, J=2.0 \mathrm{~Hz}, 1 \mathrm{H}), 5.04$ (dd, $J$ = 2.0, $7.9 \mathrm{~Hz}, 1 \mathrm{H}), 4.95(\mathrm{t}, J=7.7 \mathrm{~Hz}, 1 \mathrm{H}), 4.11(\mathrm{~s}, 2 \mathrm{H}), 3.54(\mathrm{~s}, 3 \mathrm{H}), 3.29(\mathrm{~d}, J=7.6 \mathrm{~Hz}, 1 \mathrm{H}), 2.28(\mathrm{~s}$, $3 \mathrm{H}), 1.34(\mathrm{~s}, 3 \mathrm{H}), 1.21(\mathrm{~s}, 3 \mathrm{H}) ;{ }^{13} \mathrm{C} \mathrm{NMR}\left(\mathrm{CDCl}_{3}, 200 \mathrm{MHz}\right) \delta 159.6,153.3,153.0,148.1,130.2,122.7$, $116.1,114.8,111.9,111.0,94.5,83.0,78.0,77.3,76.4,60.6,27.5,22.2,8.6$; IR (film) $v_{\max } 3462,3362$, 2984, 2937, 2839, 1807, 1707, 1636, 1595, 1497, 1387, 1371, 1331, 1263, 1169, 1109, 1078, $1036 \mathrm{~cm}^{-1}$; HRMS (ESI+) $m / z 392.1357\left(\mathrm{M}+\mathrm{H}^{+}, \mathrm{C}_{19} \mathrm{H}_{22} \mathrm{NO}_{8}\right.$ requires $\left.\mathrm{m} / \mathrm{z} 392.1346\right)$.

7

$N^{1}, N^{4}$-Bis(7-((3aR,4R,7R,7aR)-7-methoxy-6,6-dimethyl-2-oxotetrahydro-3aH-[1,3]dioxolo-

[4,5-c]pyran-4-yloxy)-8-methyl-2-oxo-2H-chromen-3-yl)terephthalamide (7). $\quad N$-(3-Dimethylaminopropyl)- $N$ '-ethylcarbodiimide hydrochloride $(26 \mathrm{mg}, 0.13 \mathrm{mmol}$ ) was added to a solution of aniline 6 (35 
$\mathrm{mg}, 90 \mu \mathrm{mol})$ and terephthalic acid $(7 \mathrm{mg}, 40 \mu \mathrm{mol})$ in $30 \%$ pyridine in $\mathrm{CH}_{2} \mathrm{Cl}_{2}(1.0 \mathrm{~mL})$. The solution was stirred for $14 \mathrm{~h}$, concentrated and the residue purified via preparative $\operatorname{TLC}\left(\mathrm{SiO}_{2}, 40: 1\right.$; $\mathrm{CH}_{2} \mathrm{Cl}_{2}$ :acetone) to afford 7 (7 mg, 19\%) as a white solid: $[\alpha]^{26}{ }_{\mathrm{D}}=-24.0^{\circ}\left(\mathrm{c}=0.15, \mathrm{CH}_{2} \mathrm{Cl}_{2}\right) ;{ }^{1} \mathrm{H} \mathrm{NMR}$ $\left(400 \mathrm{MHz}, \mathrm{CD}_{2} \mathrm{Cl}_{2}\right) \delta 8.74(\mathrm{~s}, 2 \mathrm{H}), 8.72(\mathrm{~s}, 2 \mathrm{H}), 8.00(\mathrm{~s}, 4 \mathrm{H}), 7.35$ (d, $\left.J=8.5 \mathrm{~Hz}, 2 \mathrm{H}\right), 7.10(\mathrm{~d}, J=8.5 \mathrm{~Hz}$, 2H), $5.74(\mathrm{~d}, J=1.5 \mathrm{~Hz}, 2 \mathrm{H}), 5.00(\mathrm{dd}, J=8.0,2.0 \mathrm{~Hz}, 2 \mathrm{H}), 4.90(\mathrm{t}, J=8.0 \mathrm{~Hz}, 2 \mathrm{H}), 3.50(\mathrm{~s}, 6 \mathrm{H}), 3.27$ (d, $J=8.0 \mathrm{~Hz}, 2 \mathrm{H}), 2.23(\mathrm{~s}, 6 \mathrm{H}), 1.28(\mathrm{~s}, 6 \mathrm{H}), 1.13(\mathrm{~s}, 6 \mathrm{H}) ;{ }^{13} \mathrm{C} \mathrm{NMR}\left(100 \mathrm{MHz}, \mathrm{CD}_{2} \mathrm{Cl}_{2}\right) \delta 165.1(2 \mathrm{C}), 159.4$ (2C), 155.8 (2C), 149.6 (2C), 137.5 (2C), 128.1 (4C), 126.2 (2C), 122.5 (2C), 121.7 (2C), 115.3 (2C), 114.9 (2C), 114.8 (2C), 111.7 (2C), 94.8 (2C), 82.7 (2C), 78.2 (2C), 77.5 (2C), 77.0 (2C), 60.8 (2C), 28.9 (2C), 23.2 (2C), 8.6 (2C); IR (film) $v_{\max } 3398,2984,2955,2851,1815,1715,1672,1632,1607,1529$, 1502, 1367, 1252, 1171, 1111, 1097, $1078 \mathrm{~cm}^{-1}$; HRMS $\left(\mathrm{ESI}^{+}\right.$) $\mathrm{m} / \mathrm{z} 913.2633$ (for $\mathrm{C}_{46} \mathrm{H}_{45} \mathrm{~N}_{2} \mathrm{O}_{18}, \mathrm{M}+\mathrm{H}^{+}$, exact mass requires $\mathrm{m} / \mathrm{z}$ 913.2667).

8

$N^{1}, N^{3}$-Bis(7-((3aR,4R,7R,7aR)-7-methoxy-6,6-dimethyl-2-oxotetrahydro-3aH-[1,3]dioxolo-

[4,5-c]pyran-4-yloxy)-8-methyl-2-oxo-2H-chromen-3-yl)isophthalamide (8). $\quad N$-(3-Dimethylaminopropyl)- $N$ '-ethylcarbodiimide hydrochloride $(24 \mathrm{mg}, 0.12 \mathrm{mmol}$ ) was added to a solution of aniline 6 (37 $\mathrm{mg}, 95 \mu \mathrm{mol})$ and isophthalic acid (7 mg, $43 \mu \mathrm{mol})$ in 30\% pyridine in DCM $(1.1 \mathrm{~mL})$. The solution was stirred for $14 \mathrm{~h}$, concentrated and purified via preparative TLC ( $\mathrm{SiO}_{2}, \mathrm{CH}_{2} \mathrm{Cl}_{2}$-acetone; $\left.40: 1\right)$ to afford 8 (7 mg, 41\%) as a white solid: $[\alpha]^{26}{ }_{\mathrm{D}}=-31.6^{\circ}\left(\mathrm{c}=0.50, \mathrm{CH}_{2} \mathrm{Cl}_{2}\right) ;{ }^{1} \mathrm{H} \mathrm{NMR}\left(500 \mathrm{MHz}, \mathrm{CD}_{2} \mathrm{Cl}_{2}\right) \delta 8.74(\mathrm{~s}$, 2H), 8.73 (s, 2H), 8.37 (s, 1H), $8.02(\mathrm{dd}, J=8.0,1.5 \mathrm{~Hz}, 2 \mathrm{H}), 7.61$ (dd, $J=8.0,8.0,1 \mathrm{H}), 7.35$ (d, $J=9.0$ $\mathrm{Hz}, 2 \mathrm{H}), 7.10$ (d, $J=9.0 \mathrm{~Hz}, 2 \mathrm{H}), 5.74(\mathrm{~d}, J=2.0 \mathrm{~Hz}, 2 \mathrm{H}), 5.01-4.97(\mathrm{~m}, 2 \mathrm{H}), 4.90$ (t, $J=8.5 \mathrm{~Hz}, 2 \mathrm{H})$, 3.51 (s, 6H), 3.27 (d, J = 8.0 Hz, 2H), 2.23 (s, 6H), 1.28 (s, 6H), $1.13(\mathrm{~s}, 6 \mathrm{H}) ;{ }^{13} \mathrm{C}$ NMR $(125 \mathrm{MHz}$, $\mathrm{CD}_{2} \mathrm{Cl}_{2}$ ) $\delta 165.2$ (2C), 159.4 (2C), 154.4 (2C), 153.4 (2C), 149.6 (2C), 135.1 (2C), 130.9 (2C), 129.9 (2C), 126.7 (2C), 126.3 (2C), 124.7 (2C), 122.5 (2C), 115.4 (2C), 115.0 (2C), 111.9 (2C), 94.9 (2C), $83.2(2 \mathrm{C})$, 78.4 (2C), 77.5 (2C), 76.9 (2C), 60.8 (2C), 31.0 (2C), 24.1 (2C), 8.7 (2C); IR (film) $v_{\max } 3396,3084,2984$, 2937, 2839, 1813, 1713, 1678, 1632, 1607, 1524, 1481, 1468, 1489, 1369, 1259, 1236, 1173, 1111, 1095, $1078 \mathrm{~cm}^{-1}$; HRMS $\left(\mathrm{ESI}^{+}\right.$) $\mathrm{m} / \mathrm{z} 913.2656$ (for $\mathrm{C}_{46} \mathrm{H}_{45} \mathrm{~N}_{2} \mathrm{O}_{18}, \mathrm{M}+\mathrm{H}^{+}$, exact mass requires $\mathrm{m} / \mathrm{z}$ 913.2667).

\section{9}

$N^{1}, N^{4}$-Bis(7-((3aR,4R,7R,7aR)-3,4-dihydroxy-5-methoxy-6,6-dimethyltetrahydro-2H-pyran-

2-yloxy)-8-methyl-2-oxo-2H-chromen-3-yl)terephthalamide (9). $\mathrm{Et}_{3} \mathrm{~N}(20 \mu \mathrm{L})$ was added to a solution of cyclic carbonate $7(7 \mathrm{mg}, 7.9 \mu \mathrm{mol})$ in methanol $(200 \mu \mathrm{L})$. The resulting mixture was stirred for $14 \mathrm{~h}$, and then concentrated. The residue was purified via preparative TLC $\left(\mathrm{SiO}_{2}, 10: 1 ; \mathrm{CH}_{2} \mathrm{Cl}_{2}\right.$ :methanol) to afford 9 (6 mg, 88\%) as a colorless solid: $[\alpha]^{26}{ }_{\mathrm{D}}=-90.0^{\circ}\left(\mathrm{c}=0.07,20 \%\right.$ DMSO in $\left.\mathrm{CH}_{2} \mathrm{Cl}_{2}\right) ;{ }^{1} \mathrm{H} \mathrm{NMR}$ (500 MHz, DMSO) $\delta 9.97$ (s, 2H), 8.59 (s, 2H), 8.17 (s, 4H), $7.67(\mathrm{~d}, J=8.5,2 \mathrm{H}), 7.24$ (d, J=8.5 Hz, 2H), $5.58(\mathrm{~d}, J=2.5 \mathrm{~Hz}, 2 \mathrm{H}), 5.39$ (d, $J=4.5 \mathrm{~Hz}, 2 \mathrm{H}), 5.10$ (d, $J=6.5 \mathrm{~Hz}, 2 \mathrm{H}), 4.09-3.96(\mathrm{~m}, 2 \mathrm{H}), 3.98-3.94$ 
(m, 2H), $3.56(\mathrm{~s}, 6 \mathrm{H}), 3.34(\mathrm{~d}, J=9.0 \mathrm{~Hz}, 2 \mathrm{H}), 2.15(\mathrm{~s}, 6 \mathrm{H}), 1.29$ (s, 6H), 1.09 (s, 6H); ${ }^{13} \mathrm{C} \mathrm{NMR}(125$ MHz, $\left.\mathrm{CD}_{6} \mathrm{SO}\right) \delta 165.2$ (2C), 158.0 (2C), 156.2 (2C), 149.7 (2C), 136.5 (2C), 129.9 (2C), 127.9 (4C), 126.3 (2C), $121.2(2 \mathrm{C}), 115.1(2 \mathrm{C}), 112.8(2 \mathrm{C}), 110.8$ (2C), 98.4 (2C), 83.3 (2C), $77.8(2 \mathrm{C}), 70.8(2 \mathrm{C}), 67.5(2 \mathrm{C})$, 61.1 (2C), 29.0 (2C), 22.6 (2C), 8.1 (2C); IR (film) $v_{\max } 3379,3360,2961,2930,2874,1709,1666,1607$, 1526, 1433, 1366, 1252, 1113, 1090, $1076 \mathrm{~cm}^{-1}$; HRMS (ESI ${ }^{+}$) m/z 861.3059 (for $\mathrm{C}_{44} \mathrm{H}_{49} \mathrm{~N}_{2} \mathrm{O}_{16}, \mathrm{M}+\mathrm{H}^{+}$, exact mass requires $\mathrm{m} / \mathrm{z} 861.3082$ ).

\section{0}

$N^{1}, N^{3}$-Bis(7-((2R,3R,4S,5R)-3,4-dihydroxy-5-methoxy-6,6-dimethyltetrahydro-2H-pyran-2yloxy)-8-methyl-2-oxo-2H-chromen-3-yl)isophthalamide (10). $\mathrm{Et}_{3} \mathrm{~N}(30 \mu \mathrm{L})$ was added to a solution of cyclic carbonate $8(8 \mathrm{mg}, 8.8 \mu \mathrm{mol})$ in methanol $(300 \mu \mathrm{L})$. The resulting mixture was stirred for $14 \mathrm{~h}$ and concentrated. The residue was purified via preparative $\mathrm{TLC}\left(\mathrm{SiO}_{2}, 10: 1 ; \mathrm{CH}_{2} \mathrm{Cl}_{2}\right.$ :methanol) to afford 10 (6 $\mathrm{mg}, 86 \%)$ as a colorless solid: $[\alpha]_{\mathrm{D}}^{26}=-25.7^{\circ}\left(\mathrm{c}=0.11,20 \% \mathrm{MeOH}\right.$ in $\left.\mathrm{CH}_{2} \mathrm{Cl}_{2}\right) ;{ }^{1} \mathrm{H}$ NMR $(500 \mathrm{MHz}$, $20 \% \mathrm{CD}_{3} \mathrm{OD}$ in $\mathrm{CD}_{2} \mathrm{Cl}_{2}$ ) $\delta 8.71$ (s, 2H), 8.38 (t, $J=2.0 \mathrm{~Hz}, 2 \mathrm{H}$ ), 8.05 (dd, $\left.J=8.0,2.0 \mathrm{~Hz}, 2 \mathrm{H}\right), 7.62$ (dd, $J$ $=8.0,8.0 \mathrm{~Hz}, 1 \mathrm{H}), 7.15(\mathrm{~d}, J=9.0 \mathrm{~Hz}, 2 \mathrm{H}), 5.49(\mathrm{~d}, J=2.5 \mathrm{~Hz}, 2 \mathrm{H}), 4.09-4.05(\mathrm{~m}, 2 \mathrm{H}), 3.50(\mathrm{~s}, 6 \mathrm{H})$, $3.27(\mathrm{~d}, J=9.0 \mathrm{~Hz}, 2 \mathrm{H}), 2.21(\mathrm{~s}, 6 \mathrm{H}), 1.22(\mathrm{~s}, 6 \mathrm{H}), 1.09(\mathrm{~s}, 6 \mathrm{H}) ;{ }^{13} \mathrm{C} \mathrm{NMR}\left(125 \mathrm{MHz}, 20 \% \mathrm{CD}_{3} \mathrm{OD}\right.$ in $\mathrm{CD}_{2} \mathrm{Cl}_{2}$ ) $\delta 165.9(2 \mathrm{C}), 159.7$ (2C), 156.9 (2C), 149.8 (2C), 135.0 (2C), 131.1 (2C), $129.8(2 \mathrm{C}), 126.7$ (2C), 126.3 (2C), 126.2 (2C), 121.8 (2C), 114.5 (2C), 114.0 (2C), $111.6(2 \mathrm{C}), 98.8$ (2C), $84.4(2 \mathrm{C}), 78.9$ (2C), 71.6 (2C), 68.8 (2C), 61.8 (2C), 32.3 (2C), 22.6 (2C), 9.6 (2C); IR (film) $v_{\max } 3435,3398,2976,2926$, 2853, 1711, 1666, 1632, 1605, 1520, 1367, 1261, 1238, 1134, 1092, 989, $964 \mathrm{~cm}^{-1}$; HRMS (ESI $\left.{ }^{+}\right) \mathrm{m} / \mathrm{z}$ 861.3054 (for $\mathrm{C}_{44} \mathrm{H}_{49} \mathrm{~N}_{2} \mathrm{O}_{16}, \mathrm{M}+\mathrm{H}^{+}$, exact mass requires $\mathrm{m} / \mathrm{z}$ 861.3082).

\section{5}

$N$-(7-((3aR, 4R, 7R, 7aR)-7-methoxy-6,6-dimethyl-2-oxotetrahydro-3aH-[1,3]dioxolo[4,5c]pyran-4-yloxy)-8-methyl-2-oxo-2H-chromen-3-yl)but-3-enamide (15). $\quad N$-(3-Dimethylamino-propyl)$N^{\prime}$-ethylcarbodiimide hydrochloride (39 $\mathrm{mg}, 0.205 \mathrm{mmol}$ ) was added to a solution of vinylacetic acid (14 $\mu \mathrm{L}, 14 \mathrm{mg}, 0.164 \mathrm{mmol})$ and aniline $6(32 \mathrm{mg}, 0.0818 \mathrm{mmol})$ in $\mathrm{CH}_{2} \mathrm{Cl}_{2}$ with $30 \%$ pyridine $(1 \mathrm{~mL})$. The resulting solution stirred for $14 \mathrm{~h}$, and then concentrated. The residue was purified via preparative TLC $\left(\mathrm{SiO}_{2}, 40: 1 ; \mathrm{CH}_{2} \mathrm{Cl}_{2}\right.$-acetone) to afford $15(30 \mathrm{mg}, 80 \%)$ as a colorless oil: $[\alpha]^{24}{ }_{\mathrm{D}}=-28.7^{\circ}(\mathrm{c}=1.19$, $\left.\mathrm{CH}_{2} \mathrm{Cl}_{2}\right) ;{ }^{1} \mathrm{H}$ NMR $\left(400 \mathrm{MHz}, \mathrm{CD}_{2} \mathrm{Cl}_{2}\right) \delta 8.62(\mathrm{~s}, 1 \mathrm{H}), 8.13(\mathrm{~s}, 1 \mathrm{H}), 7.36(\mathrm{~d}, J=8.7 \mathrm{~Hz}, 1 \mathrm{H}), 7.15(\mathrm{~d}, J=$ $8.7 \mathrm{~Hz}, 1 \mathrm{H}), 6.13-5.98(\mathrm{~m}, 1 \mathrm{H}), 5.81(\mathrm{~d}, J=2.1 \mathrm{~Hz}, 1 \mathrm{H}), 5.37-5.30(\mathrm{~m}, 2 \mathrm{H}), 5.10-5.05(\mathrm{~m}, 1 \mathrm{H}), 4.99$ (t, $J=7.7 \mathrm{~Hz}, 1 \mathrm{H}), 3.59$ (s, 3H), 3.36 (d, $J=7.7 \mathrm{~Hz}, 1 \mathrm{H}), 3.23$ (d, $J=7.1 \mathrm{~Hz}, 2 \mathrm{H}), 2.28$ (s, 3H), 1.38 (s, $3 \mathrm{H}), 1.20(\mathrm{~s}, 3 \mathrm{H}) ;{ }^{13} \mathrm{C}$ NMR $\left(100 \mathrm{MHz}, \mathrm{CD}_{2} \mathrm{Cl}_{2}\right) \delta 170.1,159.1,155.5,153.6,149.5,131.0,126.0,123.9$, $122.5,120.5,115.1,115.0,111.6,94.8,83.2,78.3,77.5,77.0,60.8,42.8,27.6,22.4,8.5$; IR (film) $v_{\max }$ $3331,3084,2984,2937,2849,1811,1715,1688,1609,1524,1369,1175,1111,1084,1036 \mathrm{~cm}^{-1}$; HRMS $(\mathrm{ESI}+) \mathrm{m} / \mathrm{z} 460.1586\left(\mathrm{M}+\mathrm{H}^{+}, \mathrm{C}_{23} \mathrm{H}_{26} \mathrm{NO}_{9}\right.$ requires $\left.\mathrm{m} / \mathrm{z} 460.1608\right)$. 
$N-(7-((3 a R, 4 R, 7 R, 7 \mathrm{a} R)-7-$ methoxy-6,6-dimethyl-2-oxotetrahydro-3aH-[1,3]dioxolo[4,5c]pyran-4-yloxy)-8-methyl-2-oxo-2H-chromen-3-yl)pent-4-enamide (16). $\quad N$-(3-Dimethylamino-propyl)$N$ '-ethylcarbodiimide hydrochloride $(58 \mathrm{mg}, 0.30 \mathrm{mmol})$ was added to a solution of 4-pentenoic acid (25 $\mu \mathrm{L}, 24 \mathrm{mg}, 0.24 \mathrm{mmol})$ and aniline $6(47 \mathrm{mg}, 0.120 \mathrm{mmol})$ in $\mathrm{CH}_{2} \mathrm{Cl}_{2}$ with $30 \%$ pyridine $(1.4 \mathrm{~mL})$. The resulting solution stirred for $14 \mathrm{~h}$, and then concentrated. The residue was purified via preparative TLC $\left(\mathrm{SiO}_{2}, 40: 1 ; \mathrm{CH}_{2} \mathrm{Cl}_{2}\right.$ :acetone) to afford $16(43 \mathrm{mg}, 76 \%)$ as a white, crystalline solid: $[\alpha]^{24}{ }_{\mathrm{D}}=-26.4^{\mathrm{o}}(\mathrm{c}=$ 0.36, $\left.\mathrm{CH}_{2} \mathrm{Cl}_{2}\right)$; ${ }^{1} \mathrm{H} \mathrm{NMR}\left(400 \mathrm{MHz}, \mathrm{CD}_{2} \mathrm{Cl}_{2}\right) \delta 8.57(\mathrm{~s}, 1 \mathrm{H}), 8.02(\mathrm{~s}, 1 \mathrm{H}), 7.34(\mathrm{~d}, J=8.6 \mathrm{~Hz}, 1 \mathrm{H}), 7.13(\mathrm{~d}$, $J=8.6 \mathrm{~Hz}, 1 \mathrm{H}), 5.97-5.84(\mathrm{~m}, 1 \mathrm{H}), 5.79(\mathrm{~d}, J=2.2 \mathrm{~Hz}, 1 \mathrm{H}), 5.33-5.02(\mathrm{~m}, 3 \mathrm{H}), 4.99(\mathrm{t}, J=7.8 \mathrm{~Hz}$, $1 \mathrm{H}), 3.58(\mathrm{~s}, 3 \mathrm{H}), 3.34(\mathrm{~d}, J=7.6 \mathrm{~Hz}, 1 \mathrm{H}), 2.54-2.50(\mathrm{~m}, 2 \mathrm{H}), 2.49-2.43(\mathrm{~m}, 2 \mathrm{H}), 2.27(\mathrm{~s}, 3 \mathrm{H}), 1.30(\mathrm{~s}$, 3H), $1.19(\mathrm{~s}, 3 \mathrm{H}) ;{ }^{13} \mathrm{C} \mathrm{NMR}\left(100 \mathrm{MHz}, \mathrm{CD}_{2} \mathrm{Cl}_{2}\right) \delta 172.0,159.3,155.6,153.7,149.5,137.3,126.1,123.9$, 122.7, 116.1, 115.2 (2C), 111.7, 94.9, 83.3, 78.5, 77.6, 77.1, 60.9, 37.2, 29.6, 27.8, 22.6, 8.6; IR (film) $v_{\max }$ $3331,3080,2982,2937,2841,1811,1715,1684,1632,1609,1526,1371,1259,1175,1111,1086 \mathrm{~cm}^{-1}$; HRMS (ESI+) $m / z$ 474.1788 $\left(\mathrm{M}+\mathrm{H}^{+}, \mathrm{C}_{24} \mathrm{H}_{28} \mathrm{NO}_{9}\right.$ requires $m / z$ 474.1764).

17

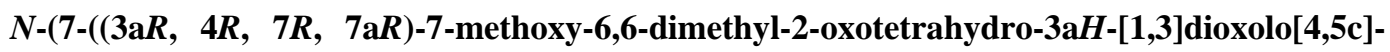
pyran-4-yloxy)-8-methyl-2-oxo-2H-chromen-3-yl)hex-5-enamide (17). $\quad N$-(3-Dimethylamino-propyl)$N$ '-ethylcarbodiimide hydrochloride $(43 \mathrm{mg}, 0.22 \mathrm{mmol})$ was added to a solution of 5-hexenoic acid (18 $\mu \mathrm{L}, 17 \mathrm{mg}, 0.148 \mathrm{mmol})$ and aniline 6 (29 mg, $0.0742 \mathrm{mmol})$ in $\mathrm{CH}_{2} \mathrm{Cl}_{2}$ with $30 \%$ pyridine $(0.9 \mathrm{~mL})$. The resulting solution stirred for $14 \mathrm{~h}$, and then the solvent was removed. The residue was purified via preparative $\mathrm{TLC}\left(\mathrm{SiO}_{2}, 40: 1 ; \mathrm{CH}_{2} \mathrm{Cl}_{2}\right.$ :acetone $)$ to afford $17(30 \mathrm{mg}, 83 \%)$ as a white foam: $[\alpha]^{24}=-27.7^{\circ}$ $\left(\mathrm{c}=1.01, \mathrm{CH}_{2} \mathrm{Cl}_{2}\right) ;{ }^{1} \mathrm{H} \mathrm{NMR}\left(400 \mathrm{MHz}, \mathrm{CD}_{2} \mathrm{Cl}_{2}\right) \delta 8.64(\mathrm{~s}, 1 \mathrm{H}), 8.00(\mathrm{~s}, 1 \mathrm{H}), 7.27(\mathrm{~d}, J=8.7 \mathrm{~Hz}, 1 \mathrm{H})$, $7.10(\mathrm{~d}, J=8.7 \mathrm{~Hz}, 1 \mathrm{H}), 5.83-5.72(\mathrm{~m}, 2 \mathrm{H}), 5.06-4.90(\mathrm{~m}, 4 \mathrm{H}), 3.58(\mathrm{~s}, 3 \mathrm{H}), 3.30(\mathrm{~d}, J=7.6 \mathrm{~Hz}, 1 \mathrm{H})$, $2.43(\mathrm{t}, J=7.5 \mathrm{~Hz} 2 \mathrm{H}), 2.26(\mathrm{~s}, 3 \mathrm{H}), 2.18-2.10(\mathrm{~m}, 2 \mathrm{H}), 1.87-1.79(\mathrm{~m}, 2 \mathrm{H}), 1.34(\mathrm{~s}, 3 \mathrm{H}), 1.14(\mathrm{~s}, 3 \mathrm{H})$;

${ }^{13} \mathrm{C}$ NMR $\left(100 \mathrm{MHz}, \mathrm{CD}_{2} \mathrm{Cl}_{2}\right) \delta 172.7,159.4,155.4,153.6,149.4,138.0,126.1,124.3,122.4,116.1,115.1$ (2C), 111.6, 94.7, 83.3, 78.3, 77.5, 77.0, 61.0, 37.2, 33.4, 27.9, 24.7, 22.6, 8.8; IR (film) $v_{\max } 3333,3078$, 2980, 2935, 2845, 1811, 1715, 1686, 1632, 1609, 1524, 1370, 1261, 1175, 1111, 1086, $1036 \mathrm{~cm}^{-1}$; HRMS (ESI+) $\mathrm{m} / \mathrm{z} 488.1898\left(\mathrm{M}+\mathrm{H}^{+}, \mathrm{C}_{25} \mathrm{H}_{30} \mathrm{NO}_{9}\right.$ requires $\left.\mathrm{m} / \mathrm{z} 474.1921\right)$.

18

$N-(7-((3 a R, 4 R, 7 R, 7 \mathrm{a} R)-7-m e t h o x y-6,6-d i m e t h y l-2-o x o t e t r a h y d r o-3 a H-[1,3] d i o x o l o[4,5 c]-$ pyran-4-yloxy)-8-methyl-2-oxo-2H-chromen-3-yl)hept-6-enamide (18). $\quad N$-(3-Dimethylamino-propyl)$N$ '-ethylcarbodiimide hydrochloride (43 mg, $0.22 \mathrm{mmol}$ ) was added to a solution of 6-heptenoic acid (24 $\mu \mathrm{L}, 23 \mathrm{mg}, 0.179 \mathrm{mmol})$ and aniline $6(35 \mathrm{mg}, 0.0895 \mathrm{mmol})$ in $\mathrm{CH}_{2} \mathrm{Cl}_{2}$ with $30 \%$ pyridine $(0.9 \mathrm{~mL})$. The 
resulting solution stirred for $14 \mathrm{~h}$, and then the solvent was removed. The residue was purified via preparative TLC $\left(\mathrm{SiO}_{2}, 40: 1 ; \mathrm{CH}_{2} \mathrm{Cl}_{2}\right.$ :acetone) to afford $18(30 \mathrm{mg}, 69 \%)$ as a white foam: $[\alpha]^{24}{ }_{\mathrm{D}}=-27.3^{\circ}$ $\left(\mathrm{c}=1.26, \mathrm{CH}_{2} \mathrm{Cl}_{2}\right) ;{ }^{1} \mathrm{H}$ NMR $\left(400 \mathrm{MHz}, \mathrm{CD}_{2} \mathrm{Cl}_{2}\right) \delta 8.67(\mathrm{~s}, 1 \mathrm{H}), 8.00(\mathrm{~s}, 1 \mathrm{H}), 7.30(\mathrm{~d}, J=8.7 \mathrm{~Hz}, 1 \mathrm{H})$, $7.15(\mathrm{~d}, J=8.7 \mathrm{~Hz}, 1 \mathrm{H}), 5.84-5.73(\mathrm{~m}, 2 \mathrm{H}), 5.08-4.92(\mathrm{~m}, 4 \mathrm{H}), 3.58(\mathrm{~s}, 3 \mathrm{H}), 3.31$ (d, $J=7.6 \mathrm{~Hz}, 1 \mathrm{H})$, $2.43(\mathrm{t}, J=7.5 \mathrm{~Hz} 2 \mathrm{H}), 2.27$ (s, 3H), $2.14-2.06(\mathrm{~m}, 2 \mathrm{H}), 1.79-1.69(\mathrm{~m}, 2 \mathrm{H}), 1.52-1.45(\mathrm{~m}, 2 \mathrm{H}), 1.35$ (s, $3 \mathrm{H}), 1.19(\mathrm{~s}, 3 \mathrm{H}) ;{ }^{13} \mathrm{C} \mathrm{NMR}\left(100 \mathrm{MHz}, \mathrm{CD}_{2} \mathrm{Cl}_{2}\right) \delta$ 172.8, 159.4, 155.4, 153.6, 149.4, 138.7, 126.1, 124.3, 122.4, 115.3, 115.1 (2C), 111.6, 94.7, 83.3, 78.3, 77.5, 77.0, 61.0, 37.9, 33.8, 28.7, 27.9, 25.2, 22.6, 8.8; IR (film) $v_{\max } 3333,3078,2980,2935,2860,1817,1813,1715,1686,1609,1524,1369,1261,1175,1111$, $1088,1036 \mathrm{~cm}^{-1}$; HRMS (ESI+) $\mathrm{m} / \mathrm{z} 502.2076\left(\mathrm{M}+\mathrm{H}^{+}, \mathrm{C}_{26} \mathrm{H}_{32} \mathrm{NO}_{9}\right.$ requires $\mathrm{m} / \mathrm{z}$ 502.2077).

\section{5a}

$N$-(7-((2R, 3R, 4S, 5R)-3,4-dihydroxy-5-methoxy-6,6-dimethyltetrahydro-2H-pyran-2-yloxy)8-methyl-2-oxo-2H-chromen-3-yl)but-3-enmaide (15a). $\mathrm{Et}_{3} \mathrm{~N}(40 \mu \mathrm{L})$ was added to a solution of cyclic carbonate $15(18 \mathrm{mg}, 39 \mu \mathrm{mol})$ in methanol $(400 \mu \mathrm{L})$. The resulting mixture was stirred for $14 \mathrm{~h}$ and then concentrated. The residue was purified via preparative $\mathrm{TLC}\left(\mathrm{SiO}_{2}, 4: 1 ; \mathrm{CH}_{2} \mathrm{Cl}_{2}\right.$ :acetone) to afford $15 \mathrm{a}$ (12 mg, 71\%) as a white solid: $[\alpha]^{24}=-33.1^{\circ}\left(\mathrm{c}=0.25,20 \% \mathrm{MeOH}\right.$ in $\left.\mathrm{CH}_{2} \mathrm{Cl}_{2}\right) ;{ }^{1} \mathrm{H} \mathrm{NMR}(400 \mathrm{MHz}, 20 \%$ $\mathrm{CD}_{3} \mathrm{OD}$ in $\left.\mathrm{CD}_{2} \mathrm{Cl}_{2}\right) \delta 8.58(\mathrm{~s}, 1 \mathrm{H}), 7.26(\mathrm{~d}, J=8.7 \mathrm{~Hz}, 1 \mathrm{H}), 7.11(\mathrm{~d}, J=8.7 \mathrm{~Hz}, 1 \mathrm{H}), 6.94-6.85(\mathrm{~m}, 1 \mathrm{H})$, $6.02(\mathrm{dd}, J=1.7,15.2 \mathrm{~Hz}, 1 \mathrm{H}), 5.46(\mathrm{~d}, J=2.2 \mathrm{~Hz}, 1 \mathrm{H}), 4.10-4.01(\mathrm{~m}, 2 \mathrm{H}), 3.49$ (s, 3H), 3.25 (dd, $J=$ 1.9, $2.6 \mathrm{~Hz}, 1 \mathrm{H}), 2.17$ (s, 3H), $1.87-1.83$ (s, 3H), 1.24 (s, 3H), 1.01 (s, 3H); ${ }^{13} \mathrm{C}$ NMR (100 MHz, 20\% $\mathrm{CD}_{3} \mathrm{OD}$ in $\left.\mathrm{CD}_{2} \mathrm{Cl}_{2}\right) \delta 163.6,157.5,154.5,147.5,141.0,124.0,123.4,123.0,120.0,112.3,112.2,109.5$, 96.7, 82.5, 76.9, 69.5, 66.7, 59.9, 26.9, 20.6, 15.9, 6.2; IR (film) $v_{\max } 3400,3323,3089,2976,2934,2834$, $1709,1680,1626,1607,1531,1445,1414,1369,1363,1084 \mathrm{~cm}^{-1}$; HRMS (ESI +$) \mathrm{m} / \mathrm{z} 434.1817\left(\mathrm{M}+\mathrm{H}^{+}\right.$, $\mathrm{C}_{22} \mathrm{H}_{28} \mathrm{NO}_{8}$ requires $\mathrm{m} / \mathrm{z}$ 434.1815).

\section{6a}

\section{$N$-(7-((2R, 3R, 4S, 5R)-3,4-dihydroxy-5-methoxy-6,6-dimethyltetrahydro-2H-pyran-2-yloxy)-}

8-methyl-2-oxo-2H-chromen-3-yl)pent-4-enmaide (16a). $\mathrm{Et}_{3} \mathrm{~N}(30 \mu \mathrm{L})$ was added to a solution of cyclic carbonate $16(13 \mathrm{mg}, 28 \mu \mathrm{mol})$ in methanol $(300 \mu \mathrm{L})$. The resulting mixture was stirred for $14 \mathrm{~h}$ and then concentrated. The residue was purified via preparative $\mathrm{TLC}\left(\mathrm{SiO}_{2}, 4: 1 ; \mathrm{CH}_{2} \mathrm{Cl}_{2}\right.$ :acetone) to afford $\mathbf{1 6 a}$ (13 mg, 81\%) as a white solid: $[\alpha]^{24}{ }_{\mathrm{D}}=-27.4^{\circ}\left(\mathrm{c}=0.22,20 \% \mathrm{MeOH}\right.$ in $\left.\mathrm{CH}_{2} \mathrm{Cl}_{2}\right) ;{ }^{1} \mathrm{H} \mathrm{NMR}(400 \mathrm{MHz}, 20 \%$ $\mathrm{CD}_{3} \mathrm{OD}$ in $\left.\mathrm{CD}_{2} \mathrm{Cl}_{2}\right) \delta 8.63(\mathrm{~s}, 1 \mathrm{H}), 8.01(\mathrm{~s}, 1 \mathrm{H}), 7.33(\mathrm{~d}, J=8.7 \mathrm{~Hz}, 1 \mathrm{H}), 7.18(\mathrm{~d}, J=8.7 \mathrm{~Hz}, 1 \mathrm{H}), 5.97-$ $5.85(\mathrm{~m}, 1 \mathrm{H}), 5.59$ (d, $J=1.8 \mathrm{~Hz}, 1 \mathrm{H}), 5.13(\mathrm{dd}, J=1.5,17.1 \mathrm{~Hz}, 1 \mathrm{H}), 5.05(\mathrm{dd}, J=1.4,10.2 \mathrm{~Hz}, 1 \mathrm{H}), 4.26$ - $4.21(\mathrm{~m}, 2 \mathrm{H}), 3.59$ (s, 3H), 3.35 (d, $J=8.7 \mathrm{~Hz}, 1 \mathrm{H}), 2.83(\mathrm{~s}, 1 \mathrm{H}), 2.76(\mathrm{~s}, 1 \mathrm{H}), 2.55-2.45(\mathrm{~m}, 4 \mathrm{H}), 2.26$ (s, 3H), $1.36(\mathrm{~s}, 3 \mathrm{H}), 1.12(\mathrm{~s}, 3 \mathrm{H}) ;{ }^{13} \mathrm{C} \mathrm{NMR}\left(100 \mathrm{MHz}, 20 \% \mathrm{CD}_{3} \mathrm{OD}\right.$ in $\left.\mathrm{CD}_{2} \mathrm{Cl}_{2}\right) \delta 171.8,159.3,156.3$, 149.4, 137.1, 125.9, 124.2, 122.1, 116.0, 114.4, 114.3, 111.4, 98.2, 84.6, 78.8, 71.6, 69.0, 62.1, 37.1, 29.5, 
29.2, 22.7, 8.4; IR (film) $v_{\max } 3398,3327,3080,2980,2928,2854,1711,1682,1607,1531,1375,1261$, 1178, 1130, $1084 \mathrm{~cm}^{-1}$; HRMS (ESI+) $\mathrm{m} / \mathrm{z} 448.1947\left(\mathrm{M}+\mathrm{H}^{+}, \mathrm{C}_{23} \mathrm{H}_{30} \mathrm{NO}_{8}\right.$ requires $\left.\mathrm{m} / \mathrm{z} 448.1971\right)$.

17a

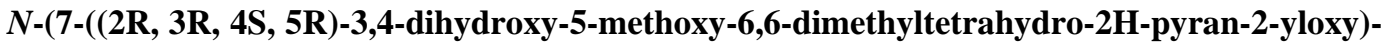
8-methyl-2-oxo-2H-chromen-3-yl)hex-5-enmaide (17a). $\mathrm{Et}_{3} \mathrm{~N}(50 \mu \mathrm{L})$ was added to a solution of cyclic carbonate $17(12 \mathrm{mg}, 25 \mu \mathrm{mol})$ in methanol $(500 \mu \mathrm{L})$. The resulting mixture was stirred for $14 \mathrm{~h}$ and then concentrated. The residue was purified via preparative TLC $\left(\mathrm{SiO}_{2}, 4: 1 ; \mathrm{CH}_{2} \mathrm{Cl}_{2}\right.$ :acetone) to afford 17a (11 mg, 96\%) as a white solid: $[\alpha]_{\mathrm{D}}^{25}=-26.1^{\mathrm{o}}\left(\mathrm{c}=0.18,20 \% \mathrm{MeOH}\right.$ in $\left.\mathrm{CH}_{2} \mathrm{Cl}_{2}\right)$; ${ }^{1} \mathrm{H} \mathrm{NMR}(400 \mathrm{MHz}$, $\left.\mathrm{CD}_{2} \mathrm{Cl}_{2}\right) \delta 8.92(\mathrm{~s}, 1 \mathrm{H}), 7.97(\mathrm{~s}, 1 \mathrm{H}), 7.33(\mathrm{~d}, J=8.7 \mathrm{~Hz}, 1 \mathrm{H}), 7.19(\mathrm{~d}, J=8.7 \mathrm{~Hz}, 1 \mathrm{H}), 5.95-5.80(\mathrm{~m}, 1 \mathrm{H})$, $5.59(\mathrm{~d}, J=1.9 \mathrm{~Hz}, 1 \mathrm{H}), 5.07$ (d, $J=17.1 \mathrm{~Hz}, 1 \mathrm{H}), 5.02(\mathrm{dd}, J=1.0,10.2 \mathrm{~Hz}, 1 \mathrm{H}), 4.26-4.20(\mathrm{~m}, 2 \mathrm{H})$, $3.59(\mathrm{~s}, 3 \mathrm{H}), 3.35(\mathrm{~d}, J=8.7 \mathrm{~Hz}, 1 \mathrm{H}), 2.74(\mathrm{~m}, 2 \mathrm{H}), 2.46-2.39(\mathrm{~m}, 2 \mathrm{H}), 2.26(\mathrm{~s}, 3 \mathrm{H}), 2.18-2.10(\mathrm{~m}, 2 \mathrm{H})$, $1.85-1.76(\mathrm{~m}, 2 \mathrm{H}), 1.36(\mathrm{~s}, 3 \mathrm{H}), 1.12(\mathrm{~s}, 3 \mathrm{H}) ;{ }^{13} \mathrm{C} \mathrm{NMR}\left(125 \mathrm{MHz}, \mathrm{CD}_{2} \mathrm{Cl}_{2}\right) \delta 172.4,159.4,156.3,149.4$, 138.2, 125.9, 124.1, 122.2, 115.6, 114.5, 114.3, 111.4, 98.2, 84.6, 78.8, 71.6, 69.0, 62.1, 37.1, 33.4, 29.3, 24.8, 22.7, 8.5; IR (film) $v_{\max } 3456,3327,3080,2978,2934,2835,1713,1682,1607,1528,1369,1263$, 1130, 1084, $991 \mathrm{~cm}^{-1}$; HRMS (ESI+) $\mathrm{m} / \mathrm{z} 462.2122\left(\mathrm{M}+\mathrm{H}^{+}, \mathrm{C}_{24} \mathrm{H}_{32} \mathrm{NO}_{8}\right.$ requires $\left.\mathrm{m} / \mathrm{z} 462.2128\right)$.

18a

$N$-(7-((2R, 3R, 4S, 5R)-3,4-dihydroxy-5-methoxy-6,6-dimethyltetrahydro-2H-pyran-2-yloxy)8-methyl-2-oxo-2H-chromen-3-yl)hept-6-enmaide (18a). $\mathrm{Et}_{3} \mathrm{~N}(30 \mu \mathrm{L})$ was added to a solution of cyclic carbonate $18(13 \mathrm{mg}, 26 \mu \mathrm{mol})$ in methanol $(300 \mu \mathrm{L})$. The resulting mixture was stirred for $14 \mathrm{~h}$ and then concentrated. The residue was purified via preparative $\mathrm{TLC}\left(\mathrm{SiO}_{2}, 4: 1 ; \mathrm{CH}_{2} \mathrm{Cl}_{2}\right.$ :acetone) to afford $18 \mathrm{a}$ (11 mg, 89\%) as a colorless solid: $[\alpha]^{25}=-27.2^{\circ}\left(\mathrm{c}=0.19,20 \% \mathrm{MeOH}\right.$ in $\left.\mathrm{CH}_{2} \mathrm{Cl}_{2}\right) ;{ }^{1} \mathrm{H} \mathrm{NMR}(400 \mathrm{MHz}$, $\left.\mathrm{CD}_{2} \mathrm{Cl}_{2}\right) \delta 8.63(\mathrm{~s}, 1 \mathrm{H}), 7.98(\mathrm{~s}, 1 \mathrm{H}), 7.33(\mathrm{~d}, J=8.7 \mathrm{~Hz}, 1 \mathrm{H}), 7.18(\mathrm{~d}, J=8.7 \mathrm{~Hz}, 1 \mathrm{H}), 5.95-5.73(\mathrm{~m}, 1 \mathrm{H})$, $5.59(\mathrm{~d}, J=1.8 \mathrm{~Hz}, 1 \mathrm{H}), 5.04(\mathrm{dd}, J=16.1,2.1 \mathrm{~Hz}, 1 \mathrm{H}), 4.97(\mathrm{dq}, J=10.2,1.1 \mathrm{~Hz}, 1 \mathrm{H}), 4.27-4.20$ (m, 2H), 3.59 (s, 3H), 3.35 (d, J=8.8 Hz, 1H), $2.79(\mathrm{~m}, 2 \mathrm{H}), 2.43$ (t, J=7.5 Hz, 2H), $2.26(\mathrm{~s}, 3 \mathrm{H}), 2.14-2.03$ $(\mathrm{m}, 2 \mathrm{H}), 1.78-1.68(\mathrm{~m}, 2 \mathrm{H}), 1.52-1.42(\mathrm{~m}, 2 \mathrm{H}), 1.36(\mathrm{~s}, 3 \mathrm{H}), 1.12(\mathrm{~s}, 3 \mathrm{H}) ;{ }^{13} \mathrm{C} \mathrm{NMR}(100 \mathrm{MHz}$ $\left.\mathrm{CD}_{2} \mathrm{Cl}_{2}\right) \delta 172.5,159.4,156.2,149.4,138.9,125.9,124.1,122.2,114.8,114.4,114.3,111.4,98.2,84.6$, 78.8, 71.6, 69.0, 62.1, 37.8, 33.8, 29.3, 28.8, 25.2, 22.6, 8.4; IR (film) $v_{\max } 3398,3329,3078,2976,2932$, 2858, 2837, 1713, 1684, 1607, 1529, 1366, 1263, 1132, 1084, 991, $966 \mathrm{~cm}^{-1}$; HRMS (ESI+) m/z 476.2266 $\left(\mathrm{M}+\mathrm{H}^{+}, \mathrm{C}_{25} \mathrm{H}_{34} \mathrm{NO}_{8}\right.$ requires $m / z$ 476.2285).

23

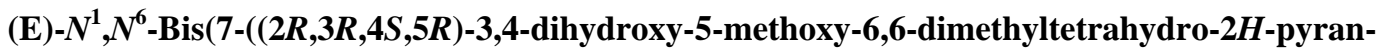
2-yloxy)-8-methyl-2-oxo-2H-chromen-3-yl)hex-3-enediamide (23). Grubbs' second generation catalyst $(11 \mathrm{mg}, .0131 \mathrm{mmol}, 10 \mathrm{~mol} \%)$ was added to a solution of olefin $15(60 \mathrm{mg}, 0.131 \mathrm{mmol})$ in $\mathrm{CH}_{2} \mathrm{Cl}_{2}(500$ 
$\mu \mathrm{L})$. The resulting solution was stirred for $14 \mathrm{~h}$, filtered through a plug of silica gel, concentrated, and purified via preparative TLC $\left(\mathrm{SiO}_{2}, 40: 1 ; \mathrm{CH}_{2} \mathrm{Cl}_{2}\right.$ :acetone) to afford dimer 19 (10mg, 9\%) that was suitable for use without further purification. Cyclic carbonate $19(10 \mathrm{mg}, .011 \mathrm{mmol})$ was dissolved in methanol containing $10 \% \mathrm{Et}_{3} \mathrm{~N}(300 \mu \mathrm{L})$ and was stirred for $14 \mathrm{~h}$. The solvent was removed, and the residue was purified via preparative TLC $\left(\mathrm{SiO}_{2}, 4: 1 ; \mathrm{CH}_{2} \mathrm{Cl}_{2}\right.$ :acetone) to afford $23(8 \mathrm{mg}, 86 \%)$ as a white solid: $[\alpha]^{25}$ $=-23.1^{\circ}(\mathrm{c}=0.07$, THF $) ;{ }^{1} \mathrm{H}$ NMR $\left(500 \mathrm{MHz}, 20 \% \mathrm{CD}_{3} \mathrm{OD}\right.$ in $\left.\mathrm{CD}_{2} \mathrm{Cl}_{2}\right) \delta 8.50(\mathrm{~s}, 2 \mathrm{H}), 7.27(\mathrm{~d}, J=9.0$ $\mathrm{Hz}, 2 \mathrm{H}), 7.12(\mathrm{~d}, J=9.0 \mathrm{~Hz}, 2 \mathrm{H}), 5.84-5.81(\mathrm{~m}, 2 \mathrm{H}), 5.46(\mathrm{~d}, J=2.0 \mathrm{~Hz}, 2 \mathrm{H}), 4.07$ (dd, $J=9.5,3.5 \mathrm{~Hz}$, 2H), 4.01 (t, $J=3.5 \mathrm{~Hz}, 2 \mathrm{H}), 3.50(\mathrm{~s}, 6 \mathrm{H}), 3.26$ (d, $J=9.5 \mathrm{~Hz}, 2 \mathrm{H}), 3.24-3.20(\mathrm{~m}, 2 \mathrm{H}), 2.17(\mathrm{~s}, 6 \mathrm{H}), 1.24$ (s, 6H), 1.01 (s, 6H); ${ }^{13} \mathrm{C}$ NMR (125 MHz, 20\% $\mathrm{CD}_{3} \mathrm{OD}$ in $\mathrm{CD}_{2} \mathrm{Cl}_{2}$ ) $\delta 171.2(2 \mathrm{C}), 159.4(2 \mathrm{C}), 156.7$ (2C), 149.6 (2C), 127.8 (2C), 126.0 (2C), 125.9 (2C), 121.8 (2C), 114.3 (2C), 114.2 (2C), $111.4(2 \mathrm{C}), 98.9$ (2C), 84.4 (2C), 78.9 (2C), 71.6 (2C), 68.7 (2C), 61.7 (2C), 46.2 (2C), 28.9 (2C), 22.5 (2C), 7.8 (2C); IR (film) $v_{\max } 3394,2964,2926,2851,1682,1605,1528,1408,1367,1356,1263,1175,1113,1086 \mathrm{~cm}^{-1}$; HRMS $\left(\mathrm{ESI}^{+}\right) \mathrm{m} / \mathrm{z} 839.3198$ (for $\mathrm{C}_{42} \mathrm{H}_{51} \mathrm{~N}_{2} \mathrm{O}_{16}, \mathrm{M}+\mathrm{H}^{+}$, exact mass requires $\mathrm{m} / \mathrm{z}$ 839.3239).

24

(E)- $N^{1}, N^{8}$-Bis(7-((2R,3R,4S,5R)-3,4-dihydroxy-5-methoxy-6,6-dimethyltetrahydro-2H-pyran2-yloxy)-8-methyl-2-oxo-2H-chromen-3-yl)oct-4-enediamide (24). Grubbs' second generation catalyst ( $4 \mathrm{mg}, 5.1 \mu \mathrm{mol}, 10 \mathrm{~mol} \%$ ) was added to a solution of olefin $16(24 \mathrm{mg}, 51 \mu \mathrm{mol})$ in $\mathrm{CH}_{2} \mathrm{Cl}_{2}(500 \mu \mathrm{L})$. The resulting solution was stirred for $14 \mathrm{~h}$, filtered through a plug of silica gel, concentrated, and purified via preparative TLC $\left(\mathrm{SiO}_{2}, 40: 1 ; \mathrm{CH}_{2} \mathrm{Cl}_{2}\right.$-acetone) to afford dimer $\mathbf{2 0}(6 \mathrm{mg}, 25 \%)$ that was suitable for use without further purification. Cyclic carbonate 20 (14 mg, $15 \mu \mathrm{mol})$ was dissolved in methanol containing $10 \% \mathrm{Et}_{3} \mathrm{~N}(300 \mu \mathrm{L})$ and was stirred for $14 \mathrm{~h}$. The solvent was removed, and the residue was purified via preparative TLC $\left(\mathrm{SiO}_{2}, 4: 1 ; \mathrm{CH}_{2} \mathrm{Cl}_{2}\right.$-acetone) to afford $24(12 \mathrm{mg}, 91 \%)$ as a white solid: $[\alpha]^{25}=-30.0^{\circ}$ (c $=0.07, \mathrm{THF}) ;{ }^{1} \mathrm{H}$ NMR $\left(400 \mathrm{MHz}, \mathrm{CD}_{6} \mathrm{SO}\right) \delta 9.55(\mathrm{~s}, 2 \mathrm{H}), 8.49(\mathrm{~s}, 2 \mathrm{H}), 7.44(\mathrm{~d}, J=8.8 \mathrm{~Hz}, 2 \mathrm{H}), 7.07$ (d, $J=8.8 \mathrm{~Hz}, 2 \mathrm{H}), 5.49-5.46(\mathrm{~m}, 2 \mathrm{H}), 5.45(\mathrm{~d}, J=2.3 \mathrm{~Hz}, 2 \mathrm{H}), 5.30$ (d, $J=4.6 \mathrm{~Hz}, 2 \mathrm{H}), 5.00$ (d, $J=6.3 \mathrm{~Hz}$, 2H), $3.96-3.94(\mathrm{~m}, 2 \mathrm{H}), 3.87-3.85(\mathrm{~m}, 2 \mathrm{H}), 3.47$ (s, 6H), 3.25 (d, J = 9.2 Hz, 2H), $2.25-2.20(\mathrm{~m}, 4 \mathrm{H})$, $2.12(\mathrm{~s}, 6 \mathrm{H}), 1.21(\mathrm{~s}, 6 \mathrm{H}), 0.99$ (s, 6H); ${ }^{13} \mathrm{C}$ NMR (125 MHz CD 6 SO) $\delta 172.1$ (2C), $157.7(2 \mathrm{C}), 155.5(2 \mathrm{C})$, 148.7 (2C), 129.4 (2C), 125.7 (2C), 125.1 (2C), 121.7 (2C), 113.2 (2C), 112.6 (2C), 110.7 (2C), $98.4(2 \mathrm{C})$, 83.3 (2C), 77.8 (2C), 70.8 (2C), 67.5 (2C), 61.1 (2C), 45.7 (2C), $35.6(2 \mathrm{C}), 29.0$ (2C), 22.9 (2C), 8.2 (2C); IR (film) $v_{\max } 3427,2953,2874,1713,1682,1605,1529,1362,1264,1184,1086 \mathrm{~cm}^{-1}$; HRMS $\left(\mathrm{ESI}^{+}\right) \mathrm{m} / \mathrm{z}$ 867.3538 (for $\mathrm{C}_{44} \mathrm{H}_{55} \mathrm{~N}_{2} \mathrm{O}_{16}, \mathrm{M}+\mathrm{H}^{+}$, exact mass requires $\mathrm{m} / \mathrm{z} 867.3552$ ).

25

(E)- $N^{1}, N^{10}$-Bis(7-((2R,3R,4S,5R)-3,4-dihydroxy-5-methoxy-6,6-dimethyltetrahydro-2Hpyran-2-yloxy)-8-methyl-2-oxo-2H-chromen-3-yl)dec-5-enediamide (25). Grubbs' second generation catalyst (6.1 mg, $7.2 \mu \mathrm{mol}, 10 \mathrm{~mol} \%$ ) was added to a solution of olefin 17 (35 mg, $72 \mu \mathrm{mol})$ in $\mathrm{CH}_{2} \mathrm{Cl}_{2}$ 
$(500 \mu \mathrm{L})$. The resulting solution was stirred for $14 \mathrm{~h}$, filtered through a plug of silica gel, concentrated, and purified via preparative TLC $\left(\mathrm{SiO}_{2}, 40: 1 ; \mathrm{CH}_{2} \mathrm{Cl}_{2}\right.$ :acetone) to afford dimer 21 (13 mg, 38\%) suitable for use without further purification. Cyclic carbonate 21 (14 mg, $15 \mu \mathrm{mol})$ was dissolved in methanol containing $10 \% \mathrm{Et}_{3} \mathrm{~N}(300 \mu \mathrm{L})$ and was stirred for $14 \mathrm{~h}$. The solvent was removed, and the residue was purified via preparative TLC $\left(\mathrm{SiO}_{2}, 4: 1 ; \mathrm{CH}_{2} \mathrm{Cl}_{2}\right.$ :acetone) to afford $25(11 \mathrm{mg}, 83 \%)$ as a white solid: $[\alpha]_{\mathrm{D}}^{25}=-19.4^{\circ}(\mathrm{c}$ $=0.17, \mathrm{THF}) ;{ }^{1} \mathrm{H} \mathrm{NMR}\left(500 \mathrm{MHz}, 20 \% \mathrm{CD}_{3} \mathrm{OD}\right.$ in $\left.\mathrm{CD}_{2} \mathrm{Cl}_{2}\right) \delta 8.52(\mathrm{~s}, 2 \mathrm{H}), 7.25(\mathrm{~d}, J=8.5 \mathrm{~Hz}, 2 \mathrm{H}), 7.10$ (d, $J=8.5 \mathrm{~Hz}, 2 \mathrm{H}), 5.45$ (d, $J=2.0 \mathrm{~Hz}, 2 \mathrm{H}), 5.39$ (s, 2H), 4.07 (dd, $J=9.0,3.5 \mathrm{~Hz}, 2 \mathrm{H}), 4.01$ (d, $J=3.5$ $\mathrm{Hz}, 2 \mathrm{H}), 3.49$ (s, 6H), 3.25-3.23 (m, 2H), 2.34 (t, J = 7.5 Hz, 4H), 2.12 (s, 6H), $2.05-1.99$ (m, 4H), $1.75-$ $1.64(\mathrm{~m}, 4 \mathrm{H}), 1.25$ (s, 6H), $1.03(\mathrm{~s}, 6 \mathrm{H}) ;{ }^{13} \mathrm{C} \mathrm{NMR}\left(125 \mathrm{MHz}, 20 \% \mathrm{CD}_{3} \mathrm{OD}\right.$ in $\left.\mathrm{CD}_{2} \mathrm{Cl}_{2}\right) \delta 173.3(2 \mathrm{C}), 159.5$ (2C), 156.5 (2C), 149.5 (2C), 130.7 (2C),126.0 (2C), 124.9 (2C), 121.9 (2C), 114.3 (2C), 114.2, 111.4 (2C), $98.7(2 \mathrm{C}), 84.5(2 \mathrm{C}), 78.9$ (2C), $71.6(2 \mathrm{C}), 68.8$ (2C), 61.9 (2C), 36.9 (2C), $32.2(2 \mathrm{C}), 28.8(2 \mathrm{C}), 25.3$ (2C), 22.6 (2C), 8.2 (2C); IR (film) $v_{\max } 3400,2918,2849,1705,1691,1661,1607,1531,1371,1261$, 1113, $1082 \mathrm{~cm}^{-1}$; HRMS $\left(\mathrm{ESI}^{+}\right.$) $\mathrm{m} / \mathrm{z} 895.3868$ (for $\mathrm{C}_{46} \mathrm{H}_{59} \mathrm{~N}_{2} \mathrm{O}_{16}, \mathrm{M}+\mathrm{H}^{+}$, exact mass requires $\mathrm{m} / \mathrm{z}$ 895.3865).

26

(E)- $N^{1}, N^{12}$-Bis(7-((2R,3R,4S,5R)-3,4-dihydroxy-5-methoxy-6,6-dimethyltetrahydro-2Hpyran-2-yloxy)-8-methyl-2-oxo-2H-chromen-3-yl)dodec-6-enediamide (26). Grubbs' second generation catalyst (4.4 mg, $5.2 \mu \mathrm{mol}, 10 \mathrm{~mol} \%$ ) was added to a solution of olefin 18 (26 mg, $52 \mu \mathrm{mol})$ in $\mathrm{CH}_{2} \mathrm{Cl}_{2}$ $(500 \mu \mathrm{L})$. The resulting solution was stirred for $14 \mathrm{~h}$, filtered through a plug of silica gel, concentrated, and purified via preparative TLC $\left(\mathrm{SiO}_{2}, 40: 1 ; \mathrm{CH}_{2} \mathrm{Cl}_{2}\right.$-acetone) to afford dimer 22 (13 $\left.\mathrm{mg}, 50 \%\right)$ that was suitable for use without further purification. Cyclic carbonate $22(13 \mathrm{mg}, 13 \mu \mathrm{mol})$ was dissolved in methanol containing $10 \% \mathrm{Et}_{3} \mathrm{~N}(300 \mu \mathrm{L})$ and was stirred for $14 \mathrm{~h}$. The solvent was removed, and the residue was purified via preparative $\mathrm{TLC}\left(\mathrm{SiO}_{2}, \mathrm{CH}_{2} \mathrm{Cl}_{2}\right.$-acetone; 4:1) to afford $\mathbf{2 6}(9 \mathrm{mg}, 76 \%)$ as a white solid: $[\alpha]^{26}{ }_{\mathrm{D}}=-41.4^{\mathrm{o}}\left(\mathrm{c}=0.07\right.$, THF); ${ }^{1} \mathrm{H}$ NMR $\left(500 \mathrm{MHz}, 20 \% \mathrm{CD}_{3} \mathrm{OD}\right.$ in $\left.\mathrm{CD}_{2} \mathrm{Cl}_{2}\right) \delta 8.50(\mathrm{~s}, 2 \mathrm{H}), 7.23$ (d, $J=9.0 \mathrm{~Hz}, 2 \mathrm{H}), 7.09$ (d, $J=9.0 \mathrm{~Hz}, 2 \mathrm{H}), 5.46(\mathrm{~d}, J=2.5 \mathrm{~Hz}, 2 \mathrm{H}), 5.39-5.32(\mathrm{~m}, 2 \mathrm{H}), 4.09-4.03(\mathrm{~m}$, 2H), 4.02 (t, $J=1.0 \mathrm{~Hz}, 2 \mathrm{H}), 3.49$ (s, 6H), 3.25 (d, $J=6.0 \mathrm{~Hz}, 2 \mathrm{H}), 2.37-2.31$ (m, 4H), 2.15 (s, 6H), 2.00 - $1.91(\mathrm{~m}, 4 \mathrm{H}), 1.71-1.61(\mathrm{~m}, 4 \mathrm{H}), 1.25(\mathrm{~s}, 6 \mathrm{H}), 1.03(\mathrm{~s}, 6 \mathrm{H}) ;{ }^{13} \mathrm{C} \mathrm{NMR}\left(125 \mathrm{MHz}, 20 \% \mathrm{CD}_{3} \mathrm{OD}\right.$ in $\mathrm{CD}_{2} \mathrm{Cl}_{2}$ ) $\delta 173.3$ (2C), 159.5 (2C), 156.5 (2C), 149.4 (2C), $130.6(2 \mathrm{C}), 125.9(2 \mathrm{C}), 124.9(2 \mathrm{C}), 121.9$ (2C), 114.4 (2C), 114.2 (2C), 111.5 (2C), 98.7 (2C), 84.5 (2C), 78.9 (2C), 71.6 (2C), 68.8 (2C), 61.9 (2C), 37.6 (2C), 32.2 (2C), 29.3 (2C), 29.1 (2C), 25.3 (2C), 22.8 (2C), 8.2 (2C); IR (film) $v_{\max } 3400,3381,3325$, 2978, 2930, 2854, 1711, 1666, 1607, 1531, 1367, 1263, 1113, $1084 \mathrm{~cm}^{-1}$; HRMS $\left(\mathrm{ESI}^{+}\right.$) $\mathrm{m} / \mathrm{z} 923.4169$ (for $\mathrm{C}_{48} \mathrm{H}_{63} \mathrm{~N}_{2} \mathrm{O}_{16}, \mathrm{M}+\mathrm{H}^{+}$, exact mass requires $m / z$ 923.4178). 
Oct-4-ynedioic acid (27). ${ }^{4,5}$ Ethyl malonate $(24.7 \mathrm{~mL}, 26.2 \mathrm{~g}, 0.164 \mathrm{~mol})$ was added over $30 \mathrm{~min}$ to a stirred suspension of sodium metal $(3.00 \mathrm{~g}, 0.130 \mathrm{~mol})$ in toluene $(150 \mathrm{~mL})$ at $50^{\circ} \mathrm{C}$. After stirring for 4 h, 1,4-dichloro-2-butyne was added dropwise and the solution commenced to stir for $14 \mathrm{~h}$ at $80^{\circ} \mathrm{C}$. The reaction mixture was cooled to $\mathrm{RT}$, washed with $\mathrm{H}_{2} \mathrm{SO}_{4}(2 \mathrm{~N}, 100 \mathrm{~mL})$, and then washed with water (100 $\mathrm{mL})$. The organic layer was dried $\left(\mathrm{Na}_{2} \mathrm{SO}_{4}\right)$, concentrated, and the residue was chromatographed $\left(\mathrm{SiO}_{2}\right.$, $600 \mathrm{~g}, 10: 1$; hexanes:ethyl acetate) to afford the tetraester $(8.6 \mathrm{~g}, 48 \%)$ as a colorless oil: ${ }^{1} \mathrm{H}$ NMR (400 MHz, DMSO) $\delta 4.18(\mathrm{qd}, J=7.0,2.0 \mathrm{~Hz}, 8 \mathrm{H}), 3.46-3.40(\mathrm{~m}, 2 \mathrm{H}), 2.67(\mathrm{dd}, J=6.8,1.6 \mathrm{~Hz}, 4 \mathrm{H}), 1.24(\mathrm{td}$, $J=7.0,1.6 \mathrm{~Hz}, 12 \mathrm{H})$. The tetraester $(7.0 \mathrm{~g}, 18.9 \mathrm{mmol})$ was added to aqueous $\mathrm{KOH}(10 \%, 40 \mathrm{~mL})$ and the resulting solution was heated under refluxed for $15 \mathrm{~h}$. After cooling to RT, the solution was acidified with $\mathrm{HCl}$ and then extracted continuously $(10 \mathrm{X})$ with ethyl acetate. The organic layer was dried $\left(\mathrm{Na}_{2} \mathrm{SO}_{4}\right)$ and concentrated to afford tetraacid $(3.7 \mathrm{~g}, 75 \%)$ as an off-white solid. The product was suitable for further use without additional purification: ${ }^{1} \mathrm{H}$ NMR (400 MHz, DMSO) $\delta 12.88(\mathrm{~s}, 4 \mathrm{H}), 3.33(\mathrm{t}, J=7.4 \mathrm{~Hz}, 2 \mathrm{H}), 2.53$ $(\mathrm{d}, J=7.4 \mathrm{~Hz}, 4 \mathrm{H})$. The tetraacid $(3.6 \mathrm{~g}, 14 \mathrm{mmol})$ was heated at $175^{\circ} \mathrm{C}$ for $45 \mathrm{~min}$ and then cooled to $\mathrm{rt}$ to furnish $27(1.7 \mathrm{~g}, 71 \%)$ as an off-white solid suitable for use without further purification: ${ }^{1} \mathrm{H}$ NMR (400 MHz, DMSO) $\delta 12.22(\mathrm{~s}, 2 \mathrm{H}), 2.38-2.32(\mathrm{~m}, 8 \mathrm{H})$.

28

Octanedioic acid (28). ${ }^{6}$ Palladium on carbon $(5 \%, 11 \mathrm{mg})$ was added to a solution of alkyne 27 $(111 \mathrm{mg}, 0.652 \mathrm{mmol})$ in ethanol $(0.7 \mathrm{~mL})$. The mixture was degassed with argon and then stirred under an atmosphere of $\mathrm{H}_{2}$ for $15 \mathrm{~h}$. The mixture was filtered through a plug of silica gel and concentrated to afford $28(100 \mathrm{mg}, 88 \%)$ as a white solid that is suitable for use without further purification: ${ }^{1} \mathrm{H} \mathrm{NMR}(400 \mathrm{MHz}$, $\left.\mathrm{CD}_{3} \mathrm{OD}\right) \delta 2.27(\mathrm{t}, J=7.4 \mathrm{~Hz}, 4 \mathrm{H}), 1.35(\mathrm{td}, \mathrm{J}=7.2,3.6 \mathrm{~Hz}, 4 \mathrm{H}), 1.64-1.56(\mathrm{~m}, 4 \mathrm{H})$.

(Z)-Oct-4-enedioic acid (29). ${ }^{7}$ Lindlar's catalyst $\left(\mathrm{Pd} / \mathrm{CaCO}_{3}, 20 \mathrm{mg}\right)$ was added to a solution of alkyne $27(195 \mathrm{mg}, 1.15 \mathrm{mmol})$ in ethanol $(1 \mathrm{~mL})$. The mixture was degassed with argon and then stirred under an atmosphere of $\mathrm{H}_{2}$ for $15 \mathrm{~h}$. The mixture was filtered through a plug of silica gel and concentrated to afford $29(197 \mathrm{mg}, 100 \%)$ as an off-white solid that is suitable for use without further purification: ${ }^{1} \mathrm{H}$ NMR (400 MHz, CD 3 OD) $\delta 5.41(\mathrm{t}, J=4.4 \mathrm{~Hz}, 2 \mathrm{H}), 2.41-2.26(\mathrm{~m}, 8 \mathrm{H})$.

30

$N^{1}, \quad N^{8}$-Bis(7-((3aR, 4R, 7R, 7aR)-7-methoxy-6,6-dimethyl-2-oxotetrahydro-3aH[1,3]dioxolo[4,5-c]pyran-4-yloxy)-8-methyl-2-oxo-2H-chromen-3-yl)oct-4-ynediamide (30). N-(3Dimethylamino-propyl)- $N$ '-ethylcarbodiimide hydrochloride $(27 \mathrm{mg}, 0.14 \mathrm{mmol})$ was added to a solution of aniline 6 (43 mg, $110 \mu \mathrm{mol})$ and diacid $27(8 \mathrm{mg}, 49 \mu \mathrm{mol})$ in 50\% pyridine in $\mathrm{CH}_{2} \mathrm{Cl}_{2}(1.1 \mathrm{~mL})$. The solution stirred for $14 \mathrm{~h}$ and then concentrated. The residue was purified via preparative $\mathrm{TLC}\left(\mathrm{SiO}_{2}, 40: 1\right.$; 
$\mathrm{CH}_{2} \mathrm{Cl}_{2}$-acetone) to afford $30(44 \mathrm{mg}, 41 \%)$ as a yellow solid: $[\alpha]^{24}=-22.9^{\circ}\left(\mathrm{c}=0.28, \mathrm{CH}_{2} \mathrm{Cl}_{2}\right) ;{ }^{1} \mathrm{H}$ NMR (400 MHz, $\left.\mathrm{CD}_{2} \mathrm{Cl}_{2}\right) \delta 8.62(\mathrm{~s}, 2 \mathrm{H}), 8.43(\mathrm{~s}, 2 \mathrm{H}), 7.32(\mathrm{~d}, J=8.8 \mathrm{~Hz}, 2 \mathrm{H}), 7.12(\mathrm{~d}, J=8.8 \mathrm{~Hz}, 2 \mathrm{H})$, $5.82(\mathrm{~d}, J=2.4 \mathrm{~Hz}, 2 \mathrm{H}), 5.11$ (dd, $J=8.0,2.4 \mathrm{~Hz}, 2 \mathrm{H}), 5.02$ (t, $J=8.0 \mathrm{~Hz}, 2 \mathrm{H}), 3.59$ (s, 6H), 3.39 (d, $J=$ $7.6 \mathrm{~Hz}, 2 \mathrm{H}), 2.66-2.60(\mathrm{~m}, 8 \mathrm{H}), 2.28(\mathrm{~s}, 6 \mathrm{H}), 1.39(\mathrm{~s}, 6 \mathrm{H}), 1.23(\mathrm{~s}, 6 \mathrm{H}) ;{ }^{13} \mathrm{C} \mathrm{NMR}\left(125 \mathrm{MHz}, \mathrm{CD}_{2} \mathrm{Cl}_{2}\right) \delta$ 170.0 (2C), 158.0 (2C), 154.3 (2C), 152.5 (2C), 148.2 (2C), 124.8 (2C), 123.1 (2C), 121.4 (2C), 113.9 (2C), 113.8 (2C), 110.4 (2C), 93.6 (2C), 82.0 (2C), 79.2 (2C), 77.2 (2C), 76.3 (2C), 75.8 (2C), 59.6 (2C), 36.0 (2C), 26.5 (2C), 21.3 (2C), 14.2 (2C), 7.3 (2C); IR (neat) $v_{\max } 3427,3408,3337,2986,2937,2841$, $1805,1711,1682,1630,1609,1537,1383,1371,1360,1259,1177,1153,1113,1088 \mathrm{~cm}^{-1}$; HRMS (ESI $\left.{ }^{+}\right)$ $\mathrm{m} / \mathrm{z} 934.3234$ (for $\mathrm{C}_{46} \mathrm{H}_{52} \mathrm{~N}_{3} \mathrm{O}_{18}, \mathrm{M}+\mathrm{NH}_{4}{ }^{+}$, exact mass requires $\mathrm{m} / \mathrm{z}$ 934.3246).

31

$N^{1}, N^{8}$-Bis(7-((2R, 3R, 4S, 5R)-3,4-dihydroxy-5-methoxy-6,6-dimethyltetrahydro-2H-pyran-2yloxy)-8-methyl-2-oxo-2H-chromen-3-yl)oct-4-ynediamide (31). $\mathrm{Et}_{3} \mathrm{~N}(30 \mu \mathrm{L})$ was added to a solution of cyclic carbonate $30(19 \mathrm{mg}, 20 \mu \mathrm{mol})$ in methanol $(300 \mu \mathrm{L})$. The resulting mixture was stirred for $14 \mathrm{~h}$ and then was concentrated. The residue was purified via preparative $\mathrm{TLC}\left(\mathrm{SiO}_{2}, \mathrm{CH}_{2} \mathrm{Cl}_{2}\right.$-methanol; 20:1) to afford $31(15 \mathrm{mg}, 85 \%)$ as a white solid: $[\alpha]^{24}{ }_{\mathrm{D}}=-16.5^{\circ}\left(\mathrm{c}=0.20\right.$, THF); ${ }^{1} \mathrm{H}$ NMR (400 MHz, DMSO) $\delta 9.69(\mathrm{~s}, 2 \mathrm{H}), 8.57(\mathrm{~s}, 2 \mathrm{H}), 7.50(\mathrm{~d}, J=9.0 \mathrm{~Hz}, 2 \mathrm{H}), 7.14(\mathrm{~d}, J=9.0 \mathrm{~Hz}, 2 \mathrm{H}), 5.52(\mathrm{~d}, J=2.0 \mathrm{~Hz}, 2 \mathrm{H})$, $5.36(\mathrm{~d}, J=4.5 \mathrm{~Hz}, 2 \mathrm{H}), 5.07(\mathrm{~d}, J=6.0 \mathrm{~Hz}, 2 \mathrm{H}), 4.05-4.00(\mathrm{~m}, 2 \mathrm{H}), 3.95-3.90(\mathrm{~m}, 2 \mathrm{H}), 3.53(\mathrm{~s}, 6 \mathrm{H})$, $3.31(\mathrm{~d}, J=9.5 \mathrm{~Hz}, 2 \mathrm{H}), 2.68(\mathrm{t}, J=6.8 \mathrm{~Hz}, 4 \mathrm{H}), 2.45(\mathrm{t}, J=6.8 \mathrm{~Hz}, 4 \mathrm{H}), 2.21(\mathrm{~s}, 6 \mathrm{H}), 1.28(\mathrm{~s}, 6 \mathrm{H}), 1.08$ (s, 6H); ${ }^{13} \mathrm{C}$ NMR (125 MHz, DMSO) $\delta 171.1$ (2C), 157.6 (2C), 155.5 (2C), 148.7 (2C), 125.7 (2C), 125.2 (2C), $121.6(2 \mathrm{C}), 113.2$ (2C), $112.6(2 \mathrm{C}), 110.7$ (2C), 98.4 (2C), 83.3 (2C), $79.6(2 \mathrm{C}), 77.8$ (2C), 70.8 (2C), 67.6 (2C), 61.1 (2C), 35.2 (2C), 28.5 (2C), 22.9 (2C), 14.2 (2C), 8.0 (2C); IR (neat) $v_{\max } 3416,2982,2935$, 1709, 1632, 1609, 1529, 1377, 1261, 1113, 1084, $991 \mathrm{~cm}^{-1} \mathrm{HRMS}\left(\mathrm{ESI}^{+}\right) \mathrm{m} / \mathrm{z} 865.3398\left(\right.$ for $\mathrm{C}_{44} \mathrm{H}_{53} \mathrm{~N}_{2} \mathrm{O}_{16}$, $\mathrm{M}+\mathrm{H}^{+}$, exact mass requires $\left.\mathrm{m} / \mathrm{z} 865.3395\right)$.

\section{2 \\ $N^{1}, \quad N^{8}$-Bis(7-((3aR, $\left.\quad 4 R, \quad 7 R, \quad 7 \mathrm{aR}\right)-7$-methoxy-6,6-dimethyl-2-oxotetrahydro-3aH-} [1,3]dioxolo[4,5-c]pyran-4-yloxy)-8-methyl-2-oxo-2H-chromen-3-yl)octanediamide (32). $\quad N$-(3Dimethylamino-propyl)- $N$ '-ethylcarbodiimide hydrochloride $(32 \mathrm{mg}, 0.17 \mathrm{mmol})$ was added to a solution of aniline 6 (50 mg, $128 \mu \mathrm{mol})$ and diacid $28(10 \mathrm{mg}, 58 \mu \mathrm{mol})$ in 50\% pyridine in $\mathrm{CH}_{2} \mathrm{Cl}_{2}(1.5 \mathrm{~mL})$. The solution stirred for $14 \mathrm{~h}$ and then was concentrated. The residue was purified via preparative TLC $\left(\mathrm{SiO}_{2}\right.$, 40:1; $\mathrm{CH}_{2} \mathrm{Cl}_{2}$-acetone) to afford $32(34 \mathrm{mg}, 29 \%)$ as a white solid: $[\alpha]^{24}{ }_{\mathrm{D}}=-28.7^{\circ}\left(\mathrm{c}=0.24, \mathrm{CH}_{2} \mathrm{Cl}_{2}\right) ;{ }^{1} \mathrm{H}$ NMR (500 MHz, $\left.\mathrm{CD}_{2} \mathrm{Cl}_{2}\right) \delta 8.54(\mathrm{~s}, 2 \mathrm{H}), 7.90(\mathrm{~s}, 2 \mathrm{H}), 7.25(\mathrm{~d}, J=8.5 \mathrm{~Hz}, 2 \mathrm{H}), 7.04(\mathrm{~d}, J=8.5 \mathrm{~Hz}, 2 \mathrm{H})$, $5.70(\mathrm{~d}, J=2.5 \mathrm{~Hz}, 2 \mathrm{H}), 4.98(\mathrm{dd}, J=8.0,2.0 \mathrm{~Hz}, 2 \mathrm{H}), 4.88(\mathrm{t}, J=8.0 \mathrm{~Hz}, 2 \mathrm{H}), 3.49$ (s, 6H), 3.25 (d, $J=$ $8.0 \mathrm{~Hz}, 2 \mathrm{H}), 2.33$ (t, J = 7.5 Hz, 4H), $2.18(\mathrm{~s}, 6 \mathrm{H}), 1.65-1.60(\mathrm{~m}, 4 \mathrm{H}), 1.36-1.30(\mathrm{~m}, 4 \mathrm{H}), 1.26(\mathrm{~s}, 6 \mathrm{H})$, $1.11(\mathrm{~s}, 6 \mathrm{H}) ;{ }^{13} \mathrm{C}$ NMR (125 MHz, $\mathrm{CD}_{2} \mathrm{Cl}_{2}$ ) $\delta 172.2(2 \mathrm{C}), 158.9$ (2C), 155.0 (2C), $153.2(2 \mathrm{C}), 149.0(2 \mathrm{C})$, 
125.6 (2C), 123.3 (2C), 122.3 (2C), 114.8 (2C), 114.7 (2C), 111.2 (2C), $94.4(2 \mathrm{C}), 82.8(2 \mathrm{C}), 78.0$ (2C), 77.2 (2C), 76.6 (2C), 60.4 (2C), 37.4 (2C), 28.8 (2C), 27.3 (2C), 25.1 (2C), 22.1 (2C), 8.1 (2C); IR (neat) $v_{\max } 3450,3394,2988,2937,2860,1809,1713,1678,1632,1609,1524,1369,1261,1175,1111,1086$, 1036, 1005, $974 \mathrm{~cm}^{-1}$ HRMS (ESI ${ }^{+}$) $\mathrm{m} / \mathrm{z} 921.3283$ (for $\mathrm{C}_{46} \mathrm{H}_{53} \mathrm{~N}_{2} \mathrm{O}_{18}, \mathrm{M}+\mathrm{H}^{+}$, exact mass requires $\mathrm{m} / \mathrm{z}$ 921.3294).

33

$N^{1}, N^{8}$-Bis(7-((2R, 3R, 4S, 5R)-3,4-dihydroxy-5-methoxy-6,6-dimethyltetrahydro-2H-pyran-2yloxy)-8-methyl-2-oxo-2H-chromen-3-yl)octanediamide (33). $\mathrm{Et}_{3} \mathrm{~N}(30 \mu \mathrm{L})$ was added to a solution of cyclic carbonate $32(19 \mathrm{mg}, 20 \mu \mathrm{mol})$ in methanol $(300 \mu \mathrm{L})$. The resulting mixture was stirred for $14 \mathrm{~h}$ and then was concentrated. The residue was purified via preparative TLC $\left(\mathrm{SiO}_{2}, 10: 1 ; \mathrm{CH}_{2} \mathrm{Cl}_{2}\right.$ :methanol) to afford 33 (9 mg, 53\%) as a white solid: $[\alpha]^{25}=-18.3^{\circ}\left(\mathrm{c}=0.12\right.$, THF); ${ }^{1} \mathrm{H}$ NMR $\left(500 \mathrm{MHz}, 30 \% \mathrm{CD}_{2} \mathrm{Cl}_{2}\right.$ in DMSO) $\delta 9.37(\mathrm{~s}, 2 \mathrm{H}), 8.57$ (s, 2H), $7.38(\mathrm{~d}, J=9.0 \mathrm{~Hz}, 2 \mathrm{H}), 7.12(\mathrm{~d}, J=9.0 \mathrm{~Hz}, 2 \mathrm{H}), 5.47$ (d, $J=2.0$ $\mathrm{Hz}, 2 \mathrm{H}), 5.20-5.16(\mathrm{~m}, 2 \mathrm{H}), 4.85-4.81(\mathrm{~m}, 2 \mathrm{H}), 4.04-4.00(\mathrm{~m}, 2 \mathrm{H}), 3.95-3.92(\mathrm{~m}, 2 \mathrm{H}), 3.51(\mathrm{~s}, 6 \mathrm{H})$, $2.46(\mathrm{t}, J=7.5 \mathrm{~Hz}, 4 \mathrm{H}), 2.20$ (s, 6H), $1.64-1.58(\mathrm{~m}, 4 \mathrm{H}), 1.36-1.30(\mathrm{~m}, 4 \mathrm{H}), 1.28(\mathrm{~s}, 6 \mathrm{H}), 1.14(\mathrm{~s}, 6 \mathrm{H})$;

${ }^{13} \mathrm{C}$ NMR (125 MHz, 30\% $\mathrm{CD}_{2} \mathrm{Cl}_{2}$ in DMSO) $\delta 169.8(2 \mathrm{C}), 154.8(2 \mathrm{C}), 152.5$ (2C), $145.6(2 \mathrm{C}), 122.2$ (2C), 121.6 (2C), 118.8 (2C), 110.2 (2C), 109.7 (2C), 107.5 (2C), 95.4 (2C), 80.4 (2C), 74.7 (2C), 67.8 (2C), 64.6 (2C), 57.9 (2C), 32.9 (2C), 25.4 (2C), 25.3 (2C), 21.9 (2C), 19.4 (2C), 4.8 (2C); IR (neat) $v_{\max }$ 3416, 2982, 2935, 1709, 1632, 1609, 1529, 1377, 1261, 1113, 1084, $991 \mathrm{~cm}^{-1}$; HRMS (ESI $\left.{ }^{+}\right) \mathrm{m} / \mathrm{z} 869.3727$ (for $\mathrm{C}_{44} \mathrm{H}_{57} \mathrm{~N}_{2} \mathrm{O}_{16}, \mathrm{M}+\mathrm{H}^{+}$, exact mass requires $\mathrm{m} / \mathrm{z}$ 869.3709).

35

(Z)- $N^{1}, N^{8}$-Bis(7-((2R,3R,4S,5R)-3,4-dihydroxy-5-methoxy-6,6-dimethyltetrahydro-2H-pyran2-yloxy)-8-methyl-2-oxo-2H-chromen-3-yl)oct-4-enediamide (35). $\quad N$-(3-Dimethylamino-propyl)- $N$ 'ethylcarbodiimide hydrochloride ( $31 \mathrm{mg}, 0.16 \mathrm{mmol}$ ) was added to a solution of aniline 6 (48 $\mathrm{mg}, 123$ $\mu \mathrm{mol})$ and diacid $29(10 \mathrm{mg}, 55 \mu \mathrm{mol})$ in $30 \%$ pyridine in $\mathrm{CH}_{2} \mathrm{Cl}_{2}(1.5 \mathrm{~mL})$. The solution stirred for $14 \mathrm{~h}$ and then was concentrated. The residue was purified via preparative TLC $\left(\mathrm{SiO}_{2}, 40: 1 ; \mathrm{CH}_{2} \mathrm{Cl}_{2}\right.$ :acetone) to afford $34(45 \mathrm{mg}, 40 \%)$ as a white solid that was suitable for use without further purification. Cyclic carbonate 34 (45 mg, $49 \mu \mathrm{mol})$ was dissolved in $\mathrm{MeOH}$ containing $10 \% \mathrm{Et}_{3} \mathrm{~N}(0.5 \mathrm{~mL})$. After stirring for $14 \mathrm{~h}$, the solvent was removed and the residue purified by preparative TLC $\left(\mathrm{SiO}_{2}, 10: 1 ; \mathrm{CH}_{2} \mathrm{Cl}_{2}\right.$ :methanol) to afford 35 (35 mg, 83\%) as a white solid: $[\alpha]^{24}{ }_{\mathrm{D}}=-24.2^{\circ}$ (c $=0.22$, THF); ${ }^{1} \mathrm{H}$ NMR (500 MHz, DMSO) $\delta 9.63(\mathrm{~s}, 2 \mathrm{H}), 8.57(\mathrm{~s}, 2 \mathrm{H}), 7.56-7.48(\mathrm{~m}, 2 \mathrm{H}), 7.22-7.16(\mathrm{~m}, 2 \mathrm{H}), 5.56(\mathrm{~d}, J=1.5 \mathrm{~Hz}, 2 \mathrm{H}), 5.46(\mathrm{t}, J=$ $4.5 \mathrm{~Hz}, 2 \mathrm{H}), 5.39-5.35(\mathrm{~m}, 2 \mathrm{H}), 5.08-5.03(\mathrm{~m}, 2 \mathrm{H}), 4.10-4.02(\mathrm{~m}, 2 \mathrm{H}), 3.95(\mathrm{~s}, 2 \mathrm{H}), 3.51(\mathrm{~s}, 6 \mathrm{H}), 3.22$ (d, $J=8.0 \mathrm{~Hz}, 2 \mathrm{H}), 2.45-2.40(\mathrm{~m}, 2 \mathrm{H}), 2.06(\mathrm{~s}, 6 \mathrm{H}), 1.25(\mathrm{~s}, 6 \mathrm{H}), 1.10(\mathrm{~s}, 6 \mathrm{H}) ;{ }^{13} \mathrm{C}$ NMR $(125 \mathrm{MHz}$, DMSO) $\delta 172.3$ (2C), 157.7 (2C), 155.5 (2C), 148.7 (2C), 129.1 (2C), 125.7 (2C), $125.1(2 \mathrm{C}), 121.8(2 \mathrm{C})$, 113.2 (2C), 112.6 (2C), 110.7 (2C), 98.4 (2C), 83.3 (2C), 77.8 (2C), 70.8 (2C), 67.6 (2C), $61.1(2 \mathrm{C}), 34.4$ 
(2C), 28.5 (2C), 22.9 (2C), 21.0 (2C), 8.00 (2C); IR (neat) $v_{\max } 3439,3404,3383,2930,2856,1711,1688$, $1645,1634,1609,1520,1371,1360,1261,1113,1084,1047,1024,991$; HRMS (ESI ${ }^{+}$) m/z 919.3138 (for $\mathrm{C}_{46} \mathrm{H}_{51} \mathrm{~N}_{2} \mathrm{O}_{18}, \mathrm{M}+\mathrm{H}^{+}$, exact mass requires $\mathrm{m} / \mathrm{z}$ 919.3137).

\section{6}

\section{$N$-(7-((3aR, 4R, 7R, 7aR)-7-methoxy-6,6-dimethyl-2-oxotetrahydro-3aH-[1,3]dioxolo[4,5-} c]pyran-4-yloxy)-8-methyl-2-oxo-2H-chromen-3-yl)pent-4-ynamide (36). $\quad N$-(3-Dimethylaminopropyl)- $N$ '-ethylcarbodiimide hydrochloride $(72 \mathrm{mg}, 0.37 \mathrm{mmol})$ was added to a solution of 1-pentynoic acid $(29 \mathrm{mg}, 0.30 \mathrm{mmol})$ and aniline $6(58 \mathrm{mg}, 0.15 \mathrm{mmol})$ in $\mathrm{CH}_{2} \mathrm{Cl}_{2}$ with $30 \%$ pyridine $(2.0 \mathrm{~mL})$. The resulting solution stirred for $14 \mathrm{~h}$, and then the solvent was removed. The residue was purified via preparative TLC $\left(\mathrm{SiO}_{2}, 40: 1 ; \mathrm{CH}_{2} \mathrm{Cl}_{2}\right.$ :acetone) to afford $36(63 \mathrm{mg}, 86 \%)$ as a yellow solid: $[\alpha]^{24}{ }_{\mathrm{D}}=-$ $26.2^{\circ}\left(\mathrm{c}=0.28, \mathrm{CH}_{2} \mathrm{Cl}_{2}\right) ;{ }^{1} \mathrm{H}$ NMR $\left(400 \mathrm{MHz}, \mathrm{CDCl}_{3}\right) \delta 8.66(\mathrm{~s}, 1 \mathrm{H}), 8.19(\mathrm{~s}, 1 \mathrm{H}), 7.38(\mathrm{~d}, J=8.6 \mathrm{~Hz}, 1 \mathrm{H})$, $7.17(\mathrm{~d}, J=8.6 \mathrm{~Hz}, 1 \mathrm{H}), 5.83$ (d, $J=2.0 \mathrm{~Hz}, 1 \mathrm{H}), 5.11$ (dd, $J=2.0,8.0 \mathrm{~Hz}, 1 \mathrm{H}), 5.01$ (t, $J=8.0 \mathrm{~Hz}, 1 \mathrm{H})$, 3.62 (s, 3H), 3.39 (d, $J=8.0 \mathrm{~Hz}, 1 \mathrm{H}), 2.73-2.67(\mathrm{~m}, 2 \mathrm{H}), 2.65-2.60(\mathrm{~m}, 2 \mathrm{H}), 2.31(\mathrm{~s}, 3 \mathrm{H}), 2.11(\mathrm{t}, J=$ $2.4 \mathrm{~Hz}, 1 \mathrm{H}), 1.39$ (s, 3h), 1.23 (s, 3H); ${ }^{13} \mathrm{C}$ NMR (125 MHz, $\left.\mathrm{CDCl}_{3}\right) \delta$ 170.1, 158.7, 155.1, 153.2, 149.0, 125.7, 123.7, 122.1, 14.7, 114.6, 111.2, 94.4, 82.8, 82.6, 77.9, 77.1, 76.6, 69.3, 60.4, 36.2, 27.2, 22.0, 14.4, 8.1; IR (neat) $v_{\max } 3439,3416,3394,2986,2937,2841,1809,1713,1682,1634,1609,1526,1340,1259$, 1173, 1111, 1086, 1036, $1005 \mathrm{~cm}^{-1}$; HRMS $\left(\mathrm{ESI}^{+}\right.$) $\mathrm{m} / \mathrm{z} 472.1607$ (for $\mathrm{C}_{24} \mathrm{H}_{26} \mathrm{~N}_{2} \mathrm{O}_{9}, \mathrm{M}+\mathrm{H}^{+}$, exact mass requires $m / z 472.1608)$.

\section{7a}

2-Azidoacetic acid (37a). ${ }^{8}$ Sodium azide $(1.3 \mathrm{~g}, 20.0 \mathrm{mmol})$ was added to a solution methyl 2bromoacetate $(1.3 \mathrm{~g}, 13.1 \mathrm{mmol})$ in DMSO $(35 \mathrm{ml})$. The mixture was stirred for $20 \mathrm{~h}$ and then poured into water $(80 \mathrm{~mL})$. The resulting solution was extracted with ether $(3 \times 50 \mathrm{~mL})$. The combined organic fractions were washed with water, followed with brine, dried with $\mathrm{Na}_{2} \mathrm{SO}_{4}$, and concentrated to afford the methyl ester $(1.5 \mathrm{~g}, 100 \%)$ as a yellow oil that was suitable for use without further purification. The residue was taken up in a THF-MeOH-water $(3: 1: 1,36 \mathrm{~mL})$ solution and $\mathrm{LiOH}(2.8 \mathrm{~g}, 65.7 \mathrm{mmol})$ was added. The resulting solution was stirred for $14 \mathrm{~h}$ and diluted with $1 \mathrm{~mL}$ of THF. The solution was acidified to $\mathrm{pH} 3$ by dropwise addition of $6 \mathrm{M} \mathrm{HCl}$. The layers were separated and the organic phase was dried $\left(\mathrm{Na}_{2} \mathrm{SO}_{4}\right)$, filtered, and concentrated to afford acid 37a $(1.1 \mathrm{~g}, 85 \%)$ as a yellow oil: ${ }^{1} \mathrm{H}$ NMR (400 $\left.\mathrm{MHz}, \mathrm{CDCl}_{3}\right) \delta 11.82(\mathrm{~s}, 1 \mathrm{H}), 4.00(\mathrm{~s}, 2 \mathrm{H})$.

\section{$38 \mathbf{a}$}

3-Azidopropanoic acid (38a). ${ }^{9}$ Glacial acetic acid $(12 \mathrm{~mL})$ was added to a solution of acrylic acid $(3.0 \mathrm{~g}, 42 \mathrm{mmol})$ and sodium azide $(10.8 \mathrm{~g}, 166 \mathrm{mmol})$ in water $(12 \mathrm{~mL})$. The resulting yellow solution was heated to $80^{\circ} \mathrm{C}$ and stirred for 3 days and 1 day at rt. The black solution was extracted with $\mathrm{CH}_{2} \mathrm{Cl}_{2}(5 \times 100 \mathrm{~mL})$ and the combined organic compounds were dried $\left(\mathrm{Na}_{2} \mathrm{SO}_{4}\right)$, filtered, and 
concentrated. The residue was purified by chromatography $\left(\mathrm{SiO}_{2}, 10: 1\right.$; hexanes:ethyl acetate) to afford 38a (2.4 g, 50\%) as a yellow oil: ${ }^{1} \mathrm{H}$ NMR (400 MHz, $\left.\mathrm{CDCl}_{3}\right) \delta 11.67(\mathrm{~s}, 1 \mathrm{H}), 3.60(\mathrm{t}, J=6.4 \mathrm{~Hz}, 2 \mathrm{H}), 2.65$ (t, $J=6.4 \mathrm{~Hz}, 2 \mathrm{H})$.

\section{7}

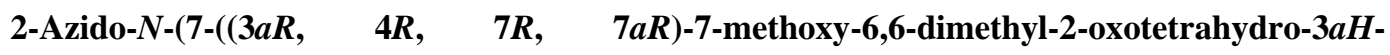
[1,3]dioxolo[4,5-c]pyran-4-yloxy)-8-methyl-2-oxo-2H-chromen-3-yl)acetamide (37). N-(3Dimethylamino-propyl)- $N$ '-ethylcarbodiimide hydrochloride $(48 \mathrm{mg}, 0.25 \mathrm{mmol}$ ) was added to a solution of azidoacid 37a (20 mg, $0.20 \mathrm{mmol}$ ) and aniline 6 (39 mg, $0.10 \mathrm{mmol})$ in $\mathrm{CH}_{2} \mathrm{Cl}_{2}$ with $30 \%$ pyridine (2.0 $\mathrm{mL}$ ). The resulting solution stirred for $14 \mathrm{~h}$, and then was concentrated. The residue was purified via preparative TLC $\left(\mathrm{SiO}_{2}, 40: 1 ; \mathrm{CH}_{2} \mathrm{Cl}_{2}\right.$ :acetone) to afford $37(40 \mathrm{mg}, 82 \%)$ as a yellow oil: $[\alpha]^{25}{ }_{\mathrm{D}}=-16.8^{\circ}$ (c = 0.19, $\left.\mathrm{CH}_{2} \mathrm{Cl}_{2}\right) ;{ }^{1} \mathrm{H}$ NMR (500 MHz, $\left.\mathrm{CD}_{2} \mathrm{Cl}_{2}\right) \delta 8.65(\mathrm{~s}, 1 \mathrm{H}), 8.62(\mathrm{~s}, 1 \mathrm{H}), 7.37(\mathrm{~d}, J=8.5 \mathrm{~Hz}, 1 \mathrm{H}), 7.15$ (d, $J=8.5 \mathrm{~Hz}, 1 \mathrm{H}), 5.81(\mathrm{~d}, J=2.5 \mathrm{~Hz}, 1 \mathrm{H}), 5.07$ (dd, $J=2.5,8.0 \mathrm{~Hz}, 1 \mathrm{H}), 4.97$ (t, $J=7.7 \mathrm{~Hz}, 1 \mathrm{H}), 4.15$ (s, 2H), 3.58 (s, 3H), 3.34 (d, J= $7.7 \mathrm{~Hz}, 1 \mathrm{H}), 2.29(\mathrm{~s}, 3 \mathrm{H}), 1.35$ (s, 3H), 1.19 (s, 3H); ${ }^{13} \mathrm{C}$ NMR $(125$ $\left.\mathrm{MHz}, \mathrm{CD}_{2} \mathrm{Cl}_{2}\right) \delta 165.4,158.2,155.1,152.8,149.0,125.5,124.1,121.0,114.5,111.0,110.9,94.1,82.4$, 77.6, 76.8, 76.3, 60.1, 26.9, 21.7, 7.8; IR (neat) $v_{\max } 3358,3319,2986,2937,2112,1809,1717,1693$, 1632, 1607, 1531, 1375, 1263, 1175, 1153, 1111, $1086 \mathrm{~cm}^{-1}$; HRMS $\left(\mathrm{ESI}^{+}\right) \mathrm{m} / \mathrm{z} 475.1447\left(\right.$ for $\mathrm{C}_{21} \mathrm{H}_{23} \mathrm{~N}_{4} \mathrm{O}_{9}$, $\mathrm{M}+\mathrm{H}^{+}$, exact mass requires $\left.\mathrm{m} / \mathrm{z} 475.1465\right)$.

\section{8

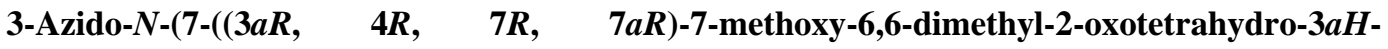 [1,3]dioxolo[4,5-c]pyran-4-yloxy)-8-methyl-2-oxo-2H-chromen-3-yl)propanamide (38). $\quad N$-(3-} Dimethylamino-propyl)- $N$ '-ethylcarbodiimide hydrochloride (46 $\mathrm{mg}, 0.24 \mathrm{mmol}$ ) was added to a solution of azidoacid 38a (22 mg, $0.19 \mathrm{mmol}$ ) and aniline 6 (37 mg, $0.095 \mathrm{mmol})$ in $\mathrm{CH}_{2} \mathrm{Cl}_{2}$ with $30 \%$ pyridine (2.0 $\mathrm{mL}$ ). The resulting solution stirred for $14 \mathrm{~h}$, and then was concentrated. The residue was purified via preparative TLC $\left(\mathrm{SiO}_{2}, 40: 1 ; \mathrm{CH}_{2} \mathrm{Cl}_{2}\right.$ :acetone) to afford $38(38 \mathrm{mg}, 79 \%)$ as a yellow solid: $[\alpha]^{25}{ }_{\mathrm{D}}=-$ $20.6^{\circ}\left(\mathrm{c}=0.65, \mathrm{CH}_{2} \mathrm{Cl}_{2}\right) ;{ }^{1} \mathrm{H}$ NMR $\left(500 \mathrm{MHz}, \mathrm{CD}_{2} \mathrm{Cl}_{2}\right) \delta 8.67(\mathrm{~s}, 1 \mathrm{H}), 8.18(\mathrm{~s}, 1 \mathrm{H}), 7.39(\mathrm{~d}, J=8.6 \mathrm{~Hz}$, $1 \mathrm{H}), 7.18(\mathrm{~d}, J=8.6 \mathrm{~Hz}, 1 \mathrm{H}), 5.84(\mathrm{~d}, J=2.0 \mathrm{~Hz}, 1 \mathrm{H}), 5.11(\mathrm{dd}, J=2.0,8.0 \mathrm{~Hz}, 1 \mathrm{H}), 5.01(\mathrm{t}, J=7.8 \mathrm{~Hz}$, 1H), 3.73 (t, $J=6.2 \mathrm{~Hz}, 2 \mathrm{H}), 3.62(\mathrm{~s}, 3 \mathrm{H}), 3.38$ (d, $J=7.8 \mathrm{~Hz}, 1 \mathrm{H}), 2.72(\mathrm{t}, J=6.2 \mathrm{~Hz}, 2 \mathrm{H}), 2.31(\mathrm{~s}, 3 \mathrm{H})$, $1.39(\mathrm{~s}, 3 \mathrm{H}), 1.23(\mathrm{~s}, 3 \mathrm{H}) ;{ }^{13} \mathrm{C}$ NMR $\left(125 \mathrm{MHz}, \mathrm{CD}_{2} \mathrm{Cl}_{2}\right) \delta 169.2,158.7,155.2,153.2,149.1,125.7,124.0$, $121.9,114.7,114.5,111.2,94.4,82.8,77.9,77.1,76.6,60.4,47.0,36.7,27.3,22.0,8.1$; IR (neat) $v_{\max }$ $3325,2984,2937,2102,1809,1713,1682,1607,1527,1384,1371,1261,1175,1111,1086 \mathrm{~cm}^{-1}$; HRMS $\left(\mathrm{ESI}^{+}\right.$) $\mathrm{m} / \mathrm{z} 489.1610$ (for $\mathrm{C}_{22} \mathrm{H}_{25} \mathrm{~N}_{4} \mathrm{O}_{9}, \mathrm{M}+\mathrm{H}^{+}$, exact mass requires $\mathrm{m} / \mathrm{z} 489.1622$ ). 


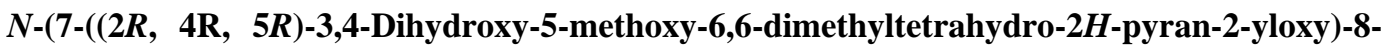
methyl-2-oxo-2H-chromen-3-yl)-3-(1-(2-(7-((2R, $3 S, \quad 4 S, \quad 5 R)-3,4 \quad$ dihydroxy-5-methoxy-6,6dimethyltetrahydro-2H-pyran-2-yloxy)-8-methyl-2-oxo-2H-chromen-3-ylamino)-2-oxoethyl)-1H1,2,3-triazol-4-yl)propanamide (41). Diisopropylethylamine (424 mg, $3.3 \mathrm{mmol}, 0.57 \mathrm{~mL}$ ) was added to a solution of alkyne 36 (32 mg, $66 \mu \mathrm{mol})$, azide 37 (32 mg, $66 \mu \mathrm{mol})$, and CuI (33 mg, $131 \mu \mathrm{mol})$ in THF $(1.0 \mathrm{~mL})$. The reaction mixture was stirred for $14 \mathrm{~h}$ at $\mathrm{rt}$ and then was concentratred. The residue was purified by preparative $\mathrm{TLC}\left(\mathrm{SiO}_{2}, 10: 1 ; \mathrm{CH}_{2} \mathrm{Cl}_{2}\right.$ :methanol) to afford cycloadduct 39 (44.2 $\left.\mathrm{mg}, 70 \%\right)$. Cyclic carbonate $39(8.6 \mathrm{mg}, 9.6 \mu \mathrm{mol})$ was dissolved in methanol $(300 \mu \mathrm{L})$ and $\mathrm{Et}_{3} \mathrm{~N}(30 \mu \mathrm{L})$ was added. The mixture was stirred for $15 \mathrm{~h}$ and then was concentrated. The residue was purified via preparative TLC $\left(\mathrm{SiO}_{2}, 10: 1 ; \mathrm{CH}_{2} \mathrm{Cl}_{2}\right.$ :methanol) to afford triazole $41(6.8 \mathrm{mg}, 85 \%)$ as a pale yellow solid: $[\alpha]_{\mathrm{D}}^{25}=-13.8^{\mathrm{o}}$ (c $=0.07,20 \% \mathrm{MeOH}$ in $\left.\mathrm{CH}_{2} \mathrm{Cl}_{2}\right) ;{ }^{1} \mathrm{H} \mathrm{NMR}\left(500 \mathrm{MHz}, 20 \% \mathrm{CD}_{3} \mathrm{OD}\right.$ in $\left.\mathrm{CD}_{2} \mathrm{Cl}_{2}\right) \delta 9.04(\mathrm{~s}, 1 \mathrm{H}), 8.97(\mathrm{~s}$, 1H), $8.23(\mathrm{~s}, 1 \mathrm{H}), 7.78(\mathrm{~d}, J=9.0 \mathrm{~Hz}, 1 \mathrm{H}), 7.72(\mathrm{~d}, J=9.0 \mathrm{~Hz}, 1 \mathrm{H}), 7.63(\mathrm{~d}, J=8.0 \mathrm{~Hz}, 2 \mathrm{H}), 6.01(\mathrm{~d}, J=$ $2.0 \mathrm{~Hz}, 1 \mathrm{H}), 6.00(\mathrm{~d}, J=2.0 \mathrm{~Hz}, 1 \mathrm{H}), 5.83(\mathrm{~s}, 1 \mathrm{H}), 4.62(\mathrm{dd}, J=2.0,9.0 \mathrm{~Hz}, 2 \mathrm{H}), 4.57(\mathrm{t}, J=2.0 \mathrm{~Hz}, 2 \mathrm{H})$, 4.05 (s, 6H), $3.81(\mathrm{~d}, J=9.0 \mathrm{~Hz}, 1 \mathrm{H}), 3.62(\mathrm{t}, J=7.0 \mathrm{~Hz}, 2 \mathrm{H}), 3.36$ (t, $J=7.0 \mathrm{~Hz}, 2 \mathrm{H}), 2.70(\mathrm{~s}, 3 \mathrm{H}), 2.69$ $(\mathrm{s}, 3 \mathrm{H}), 1.79(\mathrm{~s}, 6 \mathrm{H}), 1.56(\mathrm{~s}, 6 \mathrm{H}) ;{ }^{13} \mathrm{C} \mathrm{NMR}\left(125 \mathrm{MHz}, 20 \% \mathrm{CD}_{3} \mathrm{OD}\right.$ in $\left.\mathrm{CD}_{2} \mathrm{Cl}_{2}\right) \delta 171.5,164.6,158.6$, 158.3, 156.2, 155.8, 149.1, 148.9, 126.2, 125.4, 125.2 (2C), 123.5, 121.1, 120.6, 113.6, 113.5, 113.3, 113.0, 110.7 (2C), 98.1 (2C), 83.7 (2C), 78.2 (2C), 70.8 (2C), 68.0 (2C), 61.0 (2C), 35.6, 28.0 (2C), 21.8 (2C), 20.5, 7.3 (2C); IR (neat) $v_{\max } 3447,3421,2924,2851,1734,1697,1636,1628,1609,1558,1522,1506$, 1364, 1263, 1113, $1084 \mathrm{~cm}^{-1}$; HRMS $\left(\mathrm{ESI}^{+}\right.$) $\mathrm{m} / \mathrm{z} 894.3422$ (for $\mathrm{C}_{43} \mathrm{H}_{52} \mathrm{~N}_{5} \mathrm{O}_{16}, \mathrm{M}+\mathrm{H}^{+}$, exact mass requires $m / z$ 894.3409).

42

$N$-(7-((2R, 4S, 5R)-3,4-Dihydroxy-5-methoxy-6,6-dimethyltetrahydro-2H-pyran-2-yloxy)-8methyl-2-oxo-2H-chromen-3-yl)-3-(4-(3-(7-((2R, $\quad 4 R, \quad 5 R)-3,4-d i h y d r o x y-5-m e t h o x y-6,6-$ dimethyltetrahydro-2H-pyran-2-yloxy)-8-methyl-2-oxo-2H-chromen-3-ylamino)-3-oxopropyl)-1H-

1,2,3-triazol-1-yl)propanamide (42). Diisopropylethylamine (259 mg, $0.35 \mathrm{~mL})$ was added to a solution of alkyne 36 (19.6 mg, $40.2 \mu \mathrm{mol})$, azide 38 (23.1 mg, $60.4 \mu \mathrm{mol})$, and CuI (20 mg, $80.4 \mu \mathrm{mol})$ in THF $(0.8 \mathrm{~mL})$. The reaction mixture was stirred for $14 \mathrm{~h}$ and then was concentrated. The residue was purified by preparative $\mathrm{TLC}\left(\mathrm{SiO}_{2}, 4: 1 ; \mathrm{CH}_{2} \mathrm{Cl}_{2}\right.$ :acetone) to afford cycloadduct 40 (28.0 $\left.\mathrm{mg}, 70 \%\right)$. Cyclic carbonate $40(26.0 \mathrm{mg}, 26 \mu \mathrm{mol})$ was dissolved in methanol $(400 \mu \mathrm{L})$ and $\mathrm{Et}_{3} \mathrm{~N}(40 \mu \mathrm{L})$ was added. The reaction mixture was stirrred for $15 \mathrm{~h}$ and then was concentrated. The residue was purified via preparative TLC $\left(\mathrm{SiO}_{2}, 10: 1 ; \mathrm{CH}_{2} \mathrm{Cl}_{2}\right.$ :methanol) to afford triazole $42(20 \mathrm{mg}, 81 \%)$ as a pale yellow solid: $[\alpha]_{\mathrm{D}}^{25}=-$ $19.6^{\circ}$ (c $=0.23,20 \% \mathrm{MeOH}$ in $\left.\mathrm{CH}_{2} \mathrm{Cl}_{2}\right) ;{ }^{1} \mathrm{H} \mathrm{NMR}\left(500 \mathrm{MHz}, 20 \% \mathrm{CD}_{3} \mathrm{OD}\right.$ in $\left.\mathrm{CD}_{2} \mathrm{Cl}_{2}\right) \delta 8.78(\mathrm{~s}, 1 \mathrm{H}), 8.65$ $(\mathrm{s}, 1 \mathrm{H}), 8.56(\mathrm{~s}, 1 \mathrm{H}), 8.55(\mathrm{~s}, 1 \mathrm{H}), 7.65(\mathrm{~s}, 1 \mathrm{H}), 7.31-7.27(\mathrm{~m}, 2 \mathrm{H}), 7.19-7.14(\mathrm{~m}, 2 \mathrm{H}), 5.56(\mathrm{~d}, J=2.5$ $\mathrm{Hz}, 1 \mathrm{H}), 5.55(\mathrm{~d}, J=2.5 \mathrm{~Hz}, 1 \mathrm{H}), 4.72(\mathrm{t}, J=6.5 \mathrm{~Hz}, 1 \mathrm{H}), 4.17(\mathrm{dd}, J=3.5,9.5 \mathrm{~Hz}, 2 \mathrm{H}), 4.12(\mathrm{t}, J=2.0$, $2 \mathrm{H}), 3.62(\mathrm{~s}, 3 \mathrm{H}), 3.59(\mathrm{~s}, 3 \mathrm{H}), 3.33(\mathrm{~m}, 2 \mathrm{H}), 3.20-3.05(\mathrm{~m}, 4 \mathrm{H}), 2.85(\mathrm{t}, J=6.5 \mathrm{~Hz}, 2 \mathrm{H}), 2.24(\mathrm{~s}, 6 \mathrm{H}), 1.34$ 
(s,6H), $1.01(\mathrm{~s}, 6 \mathrm{H}) ;{ }^{13} \mathrm{C}$ NMR $\left(125 \mathrm{MHz}, 20 \% \mathrm{CD}_{3} \mathrm{OD}\right.$ in $\left.\mathrm{CD}_{2} \mathrm{Cl}_{2}\right) \delta$ 171.6, 169.1, 158.9, 158.7, 156.2, 156.0, 149.1, 149.0, 146.1, 125.6, 125.5, 125.4, 125.1, 122.6, 121.3, 121.1, 113.9, 113.8, 113.6, 113.4, 111.0, 110.9, 98.3 (2C), 84.0, 78.4, 71.1, 71.0, 68.3, 63.2, 45.5, 36.6, 35.9, 28.4, 28.3, 22.1 (2C), 20.8, 7.7 (2C); IR (neat) $v_{\max } 3439,3421,3393,2986,2932,1634,1609,1524,1367,1261,1113,1084,991 \mathrm{~cm}^{-1}$; HRMS (ESI ${ }^{+}$) $m / z 908.3571$ (for $\mathrm{C}_{44} \mathrm{H}_{54} \mathrm{~N}_{5} \mathrm{O}_{16}, \mathrm{M}+\mathrm{H}^{+}$, exact mass requires $m / z$ 908.3566).

References:

1. Yu, X. M.; Shen, G.; Neckers, L.; Blake, H.; Holzbeierlein, J.; Cronk, B.; Blagg, B. S. J., Hsp90 Inhibitors Identified from a Library of Novobiocin Analogues. J. Amer. Chem. Soc. 2005, 127, (37), 12778-12779.

2. Shen, G.; Yu, X. m.; Blagg, B. S. J., Syntheses of photolabile novobiocin analogues. Bioorg. \& Med. Chem. Let. 2004, 14, (23), 5903-5906.

3. Toplak, R.; Svete, J.; Stanovnik, B.; Grdadolnik, S. G., The synthesis of methyl 2(benzyloxycarbonyl)amino-3-dimethylaminopropenoate. The synthesis of trisubstituted pyrroles, 3-amino-2H-pyran-2-ones, fused 2H-pyran-2-ones and 4H-pyridin-4-ones. J. Heterocyclic Chem. 1999, 36, (1), 225-235.

4. Jones, E.; Mansfield, G.; Whiting M. Researches on acetylenic compounds. Part XLVII. The prototropic rearrangements of some acetylenic dicarboxylic acids. J. Chem. Soc. 1954, 3209-3212.

5. Johnson, A. 2-Butyne-1:4-diol. Part I. Reactions of the hydroxyl groups. J. Chem. Soc. 1946, 1009-1014.

6. Das, S.; Chaubey, G.; Mahanti, M. Kinetics and mechanism of the oxidative cleavage of cyclic ketones by quinolinium dichromate. Oxidative Commun. 2004, 27, (2), 349-355.

7. Manzocchi, A.; Astori, F.; Santaniello, E. A convenient synthesis of 4-oxobutanoic acid from 1,5cyclooctadiene. Synthesis. 1983, (4), 324-325.

8. Carboni, B.; Vaultier, M.; Carrie, R. Chemoselectivity of the reaction of dichloroboranes with azides: an efficient synthesis of secondary amines. Tetrahedron. 1987, 43, (8), 1799-1810.

9. Lakanen, J.; Coward, J.; Pegg, A. $\alpha$-Methyl polyamines: metabolically stable spermidine and spermine mimics capable of supporting growth in cells depleted of polyamines. J. Med. Chem. 1992, 35, 724-734. 